\title{
Four-component relativistic time-dependent density-functional theory using a stable noncollinear DFT ansatz applicable to both closed- and open-shell systems
}

\author{
Stanislav Komorovsky, ${ }^{1, a)}$ Peter J. Cherry, ${ }^{1}$ and Michal Repisky ${ }^{2}$ \\ ${ }^{1)}$ Institute of Inorganic Chemistry, Slovak Academy of Sciences, Dúbravská cesta 9, SK-84536 Bratislava, \\ Slovakia \\ ${ }^{2)}$ Hylleraas Centre for Quantum Molecular Sciences, Department of Chemistry, UiT-The Arctic University of Norway, \\ Tromsø, Norway
}

(Dated: 18 October 2019)

\begin{abstract}
We present a formulation of relativistic linear response time-dependent density functional theory for calculation of electronic excitation energies in the framework of the four-component Dirac-Coulomb Hamiltonian. This approach is based on the noncollinear ansatz originally developed by Scalmani and Frisch [J. Chem. Theory Comp. 8, 2193 (2012)], and improves upon past treatment of the limit cases in which the spin density approaches zero. As a result of these improvements, the presented approach is capable of treating both closed- and open-shell reference states. Robust convergence of the Davidson-Olsen eigenproblem algorithm for open-shell reference states was achieved through the use of a solver which considers both left and right eigenvectors. The applicability of the present methodology on both closed- and open-shell reference states is demonstrated on calculations of low-lying excitation energies for Group 3 atomic systems $\left(\mathrm{Sc}^{3+}-\mathrm{Ac}^{3+}\right)$ with non-degenerate ground states, as well as for Group 11 atomic systems $(\mathrm{Cu}-\mathrm{Rg})$ and octahedral actinide complexes $\left(\mathrm{PaCl}_{6}^{2-}, \mathrm{UCl}_{6}^{-}\right.$, and $\left.\mathrm{NpF}_{6}\right)$ with effective doublet ground states.
\end{abstract}

\section{INTRODUCTION}

Linear response time-dependent density functional theory (LR-TDDFT) is a widely used technique for calculation of electronic excitation energy spectra,${ }^{1-9}$ due to its favorable balance between accuracy and computational efficiency. However, approaches to LR-TDDFT which incorporate relativistic spin-orbit coupling effects variationally have seen comparatively modest development. This is steadily changing due to the increasing prominence of a number of areas in which more accurate description of relativistic phenomena is desirable. Such areas include X-ray spectroscopy, phosphorescence of organometallic complexes, zero-field-splitting, and other spin-related phenomena in magnetic materials. ${ }^{10-17}$

A key reason for the relatively low usage of relativistic LR-TDDFT methods is the technical challenge posed by their efficient implementation, particularly if they are intended to be applied to openshell species. Nonetheless, computationally efficient implementations of two-component LR-TDDFT, featuring variational inclusion of spin-orbit coupling (SOC) effects, and which are capable of calculating phosphorescence lifetimes of organometallic compounds, have recently been presented. ${ }^{18,19}$ Furthermore, the present authors have demonstrated that a four-component methodology is applicable to systems with up to 100 atoms, for calculation of nuclear magnetic resonance and electron paramagnetic resonance parameters. ${ }^{20-24}$

Relativistic approaches to LR-TDDFT can be loosely classified using a combination of two criteria. The

a)Electronic mail: stanislav.komorovsky@savba.sk first criterion is the method by which SOC and other relativistic effects are accounted for. These range from highly accurate methods employing four-component Dirac-Coulomb and Dirac-Coulomb-Breit Hamiltonians, more approximate methods based on two-component Hamiltonians, to methods in which SOC is incorporated using perturbation theory. The former of these are more rigorous and costly techniques well suited to precise description of systems featuring strong relativistic effects, whilst the latter are more computationally efficient, albeit more approximate, methods suited to treatment of larger systems, and those not requiring accurate description of relativistic phenomena. The second criterion by which LR-TDDFT methods which include SOC may be classified is the approach taken to ensure the rotational invariance of the DFT functional. This is typically accomplished either through use of a fully noncollinear DFT functional, which in general depends on gradients of the spin magnetization, or through use of the adiabatic local density approximation (ALDA $)^{25-27}$. The latter approach is more approximate, as it uses ALDA kernel regardless of the type of functional used to compute the ground-state wave function, yet its numerical stability means it is currently the more popular of the two. Methods of all types often employ the Tamm-Dancoff approximation (TDA), ${ }^{28}$ which increases the robustness of the eigenproblem solvers (EPS), albeit at the expense of computational accuracy.

Major developments in the field of relativistic LRTDDFT methods featuring variational inclusion of SOC began with the independent works of Gao et al. ${ }^{29}$ in the four-component framework, and Wang and Ziegler ${ }^{30}$ in the two-component framework. Both works combine noncollinear DFT theory with the ALDA approximation. 
The current status of the field is described in the review of Liu and $\mathrm{Xiao}^{31}$, where the interested reader can find comprehensive discussion of currently available relativistic LR-TDDFT methods. Three of these methods are of particular relevance to the current article, and merit further discussion. Bast et al. ${ }^{32}$ presented LR-TDDFT theory for closed-shell reference states in the framework of the Dirac-Coulomb Hamiltonian with a full noncollinear DFT kernel. The present authors consider this to be the preferred method when starting from a closed-shell reference state. Other LR-TDDFT methods that include SOC variationally and only consider closed-shell reference states introduce either minor improvements at the four-component level of theory, or use approximate two-component Hamiltonians to gain, e.g., higher computational efficiency. ${ }^{33} \mathrm{Li}$ et al. ${ }^{34}$ made an important step forward by taking into consideration open-shell reference states. In this method, termed sf-X2C-S-TDASOC, many-electron states are first obtained by the LRTDDFT based on a scalar-relativistic Hamiltonian, with subsequent diagonalization of the SOC operator over the basis of these states. A major advantage of this approach is that it guarantees full spin symmetry in the first stage of the calculation. Drawbacks of this method are the use of the ALDA kernel for important spin-flip transitions, the use of the TDA approximation when treating open-shell species, and the fact that this method is not suited to systems which exhibit strong SOC effects since it includes SOC non-variationally. Finally, Egidi et al. ${ }^{35}$ presented an elegant method based on a two-component Hamiltonian, applicable to both closed- and open-shell reference states, and utilizing a fully noncollinear DFT functional. However, at the time of writing, the numerical results of this method for prediction of electronic spectra of systems with open-shell reference states has only been demonstrated on $\mathrm{UO}_{2}^{2+}$; a system for which the ground state is considered non-degenerate ${ }^{32,36-39}$ with a single-determinant Kramers-restricted configuration (see further discussion in Sec. IV). It is worth noting that whilst there has been significant progress on relativistic LR-TDDFT as applied to closed-shell reference states, progress with regards to open-shell states remains limited, with the exception of the above discussed works. ${ }^{34,35}$

Many of the challenges associated with noncollinear DFT methods stem from the need to preserve the rotational invariance of results following the introduction of SOC terms into the Hamiltonian. Ideally, this would be accomplished through the use of genuine noncollinear DFT functionals, which satisfy this requirement by virtue of their mathematical definition. Some work has already been done to this end. ${ }^{40-43}$ Of particular note is the work of Tellgren, ${ }^{43}$ where a noncollinear variable, $g_{s s}[$ Eq. (18)], appears in an expression for the lower bound of the kinetic energy density. This is an important step towards rigorous noncollinear generalized gradient approximation (GGA) functionals, and also partially validates the a posteriori definition of noncollinear variables found in the method of Scalmani and Frisch. ${ }^{44}$ In this second approach to noncollinear DFT, the variables used in collinear DFT functionals are replaced by their noncollinear counterparts. Whilst not entirely rigorous, until a genuine noncollinear functional is developed and validated, the use of such noncollinear variables is the most practical option from an implementation perspective. Early works in this direction can be found in articles by Kubler et al. ${ }^{45}$, Sandratskii ${ }^{46}$, and van Wüllen ${ }^{47}$, in which the theory of noncollinear LDA functionals was developed. Unfortunately, attempts to directly extend this approach from LDA to GGA-based functionals have thus far been frustrated by numerical instabilities. ${ }^{31,44}$ Scalmani and Frisch ${ }^{44}$ resolved many of these instabilities through an elegant noncollinear ansatz, which introduces rotationally invariant variables for GGA functionals. Moreover, this ansatz allows for nonzero local torque on the spin magnetization, whilst satisfying the global zero-torque theorem. ${ }^{48}$ On the other hand, this approach does not adequately distinguish between the tranverse and longitudinal spin density gradients, the significance of which is discussed at length in Ref. ${ }^{41}$.

In this work we aim to predict excitation energies using a method which includes relativistic effects variationally, allowing us to treat systems containing elements across the periodic table. This goal is achieved through the use of a four-component linear response time-dependent density functional theory approach based on a Kramersunrestricted reference state (4c-LR-KU-TDDFT), combined with the noncollinear methodology proposed by Scalmani and Frisch. ${ }^{44}$ As such, the method is fully relativistic by design, is applicable to both closed- and openshell reference states, and inherently capable of describing spin-flip and spin-forbidden transitions. An interesting consequence of this approach is the method's prediction of the first excitation energy of an effective doublet system, which is zero due to the energetic degeneracy of the Kramers pair. Another illustrative consequence is that in the case of closed-shell systems the method yields non-zero singlet-triplet transition dipole moments (which correspond to spin-flip transitions, see Ref. 49), thus describing the physical mechanism responsible for phosphorescence.

In the following, Sec. II begins with a summary of the theoretical background of the new four-component linear response Kramers-unrestricted TDDFT method (Sec. II A), followed by a formulation of a noncollinear exchange-correlation potential and kernel (Sec. IIB), and definitions of its behavior in important limit cases (Sec. IIC). The section concludes with a presentation of an improved Davidson-Olsen eigenproblem solver (Sec. IID), where both left and right eigenvectors are used to construct the trial subspace. Sec. III contains computational details of a number of calculations used to illustrate possible applications of the method. Results of these calculations are discussed in Sec. IV. 


\section{THEORY AND IMPLEMENTATION}

In this work, indexes obey the following conventions: $i, j$ denote occupied orbitals, $a, b$ unoccupied positiveand negative-energy orbitals, $p$ all positive- and negativeenergy orbitals, and $k, l$ Cartesian indices. Greek letters, $\mu, \nu, \lambda, \tau$, are flattened four-component atomic orbital basis indices, which are employed to reduce the indexation complexity arising from the multicomponent nature of the spinor basis. Each flattened index specifies a scalar basis function, $\chi_{\eta}$, whilst also encoding the four-by-four matrix by which $\chi_{\eta}$ is multiplied as a consequence of the restricted kinetic balance (RKB) condition ${ }^{50,51}$. Accordingly, the $\mu$ th atomic orbital function, $\mathbf{X}_{\mu}^{\mathrm{RKB}}$, corresponds to a vector with four components

$$
X_{m, \mu}^{\mathrm{RKB}}=X_{m, n \eta}^{\mathrm{RKB}}=\left(\begin{array}{cc}
1 & 0 \\
0 & \frac{1}{2 c} \vec{\sigma} \cdot \vec{p}
\end{array}\right)_{m n} \chi_{\eta},
$$

where $\mu$ is a flattened index, $\mu=n \eta, c$ is the speed of light, $\vec{p}$ represents the momentum operator, $m, n=$ $1 \ldots 4, \chi_{\eta}$ are scalar atomic orbitals (Gaussian-type functions in this work), and $\vec{\sigma}$ is a vector defined by three Pauli matrices

$\sigma_{1}=\left(\begin{array}{cc}0 & 1 \\ 1 & 0\end{array}\right), \quad \sigma_{2}=\left(\begin{array}{cc}0 & -i \\ i & 0\end{array}\right), \quad \sigma_{3}=\left(\begin{array}{cc}1 & 0 \\ 0 & -1\end{array}\right)$.

Atomic units are used throughout this work, and summation over repeated indices is assumed unless stated otherwise. Bold font indicates either a matrix or a vector quantity, depending on the context.

\section{A. Four-component linear response Kramers-unrestricted TDDFT theory (4c-LR-KU-TDDFT)}

The LR-TDDFT and LR-TDHF theories are wellestablished in the literature,${ }^{1}$ hence only a brief overview is included here. To calculate vertical excitation energies in the framework of the LR-TDDFT or LR-TDHF theories one needs to solve the following eigenvalue equation

$$
\left(\begin{array}{cc}
\mathbf{A} & \mathbf{B} \\
-\mathbf{B}^{*} & -\mathbf{A}^{*}
\end{array}\right)\left(\begin{array}{c}
\mathbf{X} \\
\mathbf{Y}
\end{array}\right)=\omega\left(\begin{array}{c}
\mathbf{X} \\
\mathbf{Y}
\end{array}\right)
$$

where $\omega$ represents an excitation energy, $|\mathbf{X ~ Y}\rangle$ is a transition vector, and matrices $\mathbf{A}$ and $\mathbf{B}$ have the following structure

$$
\begin{gathered}
A_{a i, b j}=\left(\varepsilon_{a}-\varepsilon_{i}\right) \delta_{a b} \delta_{i j}+K_{\mu \nu, \lambda \tau} C_{\mu a}^{*} C_{\nu i} C_{\lambda j}^{*} C_{\tau b}, \\
B_{a i, b j}=K_{\mu \nu, \lambda \tau} C_{\mu a}^{*} C_{\nu i} C_{\lambda b}^{*} C_{\tau j} \\
K_{\mu \nu, \lambda \tau}(\xi)=K_{\mu \nu, \lambda \tau}^{\mathrm{hf}}(\xi)+K_{\mu \nu, \lambda \tau}^{\mathrm{xc}}(\xi) .
\end{gathered}
$$

Molecular orbital coefficients, $C_{\mu p}$, and one-electron energies, $\varepsilon_{p}$, are solutions of the Dirac-Fock equation (the summation over index $p$ is not assumed)

$$
\begin{gathered}
{\left[h_{\mu \nu}^{D}+V_{\mu \nu}^{\mathrm{hf}}(\xi)+V_{\mu \nu}^{\mathrm{xc}}(\xi)\right] C_{\nu p}=\varepsilon_{p} S_{\mu \nu} C_{\nu p},} \\
h_{\mu \nu}^{D}=\left\langle\mathbf{X}_{\mu}^{\mathrm{RKB}}\left|c \overrightarrow{\boldsymbol{\alpha}} \cdot \vec{p}+c^{2} \boldsymbol{\beta}^{\prime}+\mathbf{V}^{\mathrm{nuc}}\right| \mathbf{X}_{\nu}^{\mathrm{RKB}}\right\rangle, \\
S_{\mu \nu}=\left\langle\mathbf{X}_{\mu}^{\mathrm{RKB}} \mid \mathbf{X}_{\nu}^{\mathrm{RKB}}\right\rangle,
\end{gathered}
$$

where $\mathbf{V}^{\text {nuc }}$ is the nuclear-electron electrostatic potential, with the nuclear charge distribution represented by a Gaussian function, ${ }^{52}$ and $\overrightarrow{\boldsymbol{\alpha}}$ and $\boldsymbol{\beta}^{\prime}$ are four-component matrices of form

$$
\overrightarrow{\boldsymbol{\alpha}}=\left(\begin{array}{cc}
\mathbf{0} & \overrightarrow{\boldsymbol{\sigma}} \\
\overrightarrow{\boldsymbol{\sigma}} & \mathbf{0}
\end{array}\right), \quad \boldsymbol{\beta}^{\prime}=-2\left(\begin{array}{cc}
\mathbf{0} & \mathbf{0} \\
\mathbf{0} & \mathbf{1}
\end{array}\right) .
$$

The scalar parameter, $\xi$, weights the exact-exchange with the DFT exchange-correlation (xc) contribution. The components of the two-electron Hartree-Fock (hf) potential, $\mathbf{V}^{\mathrm{hf}}$, and kernel, $\mathbf{K}^{\mathrm{hf}}$, may be expressed as

$$
\begin{array}{r}
V_{\mu \nu}^{\mathrm{hf}}(\xi)=K_{\mu \nu, \lambda \tau}^{\mathrm{hf}}(\xi) D_{\tau \lambda}, \quad D_{\tau \lambda}=C_{\tau i} C_{\lambda i}^{*}, \\
K_{\mu \nu, \lambda \tau}^{\mathrm{hf}}(\xi)=\iint\left(\frac{\Omega_{\mu \nu}^{0}\left(\vec{r}_{1}\right) \Omega_{\lambda \tau}^{0}\left(\vec{r}_{2}\right)}{\left|\vec{r}_{1}-\vec{r}_{2}\right|}\right. \\
\left.-\xi \frac{\Omega_{\mu \tau}^{0}\left(\vec{r}_{1}\right) \Omega_{\lambda \nu}^{0}\left(\vec{r}_{2}\right)}{\left|\vec{r}_{1}-\vec{r}_{2}\right|}\right) \mathrm{dV}_{1} \mathrm{dV} V_{2},
\end{array}
$$

where $\vec{r}$ is an electron position vector, and the overlap distribution matrix has the form

$$
\Omega_{\mu \nu}^{0}=\left(X_{m, \mu}^{\mathrm{RKB}}\right)^{*} X_{m, \nu}^{\mathrm{RKB}} .
$$

The noncollinear exchange-correlation potential, $\mathbf{V}^{\mathrm{xc}}$, and kernel, $\mathbf{K}^{\mathrm{xc}}$, will be defined in the next section.

\section{B. Noncollinear DFT functionals}

In one-component (1c) theories (non-relativistic or scalar relativistic) spin is a good quantum number, hence the choice of orientation of the spin quantization axis is arbitrary. Consequently, the spin-polarized GGA density functional theory is equivalently parametrized either in terms of $\alpha$ and $\beta$ spin densities, $\rho^{\alpha}$ and $\rho^{\beta}$, or in terms of the charge and the $z$-component of spin density, $\rho_{0}$ and $\rho_{z}$,

$$
\begin{array}{ll}
\rho_{0}^{1 c}=\rho^{\alpha}+\rho^{\beta}, & \vec{\nabla} \rho_{0}^{1 c}=\vec{\nabla} \rho^{\alpha}+\vec{\nabla} \rho^{\beta}, \\
\rho_{z}^{1 c}=\rho^{\alpha}-\rho^{\beta}, & \vec{\nabla} \rho_{z}^{1 c}=\vec{\nabla} \rho^{\alpha}-\vec{\nabla} \rho^{\beta} .
\end{array}
$$

The exchange-correlation energy is then defined as

$$
E^{\mathrm{xc}}=\int \varepsilon^{\mathrm{xc}}\left[n, s, g_{n n}, g_{s s}, g_{n s}\right] \mathrm{dV},
$$

where the $\varepsilon^{\mathrm{xc}}$ denotes the exchange-correlation energy density. To ensure rotational invariance of the total energy in the framework of $1 \mathrm{c}$ theories, instead of using the 
parameters defined in Eqs. (14) and (15) the following set of parameters is employed ${ }^{53}$

$$
\begin{aligned}
n^{1 c} & =\rho_{0}^{1 c}, \\
s^{1 c} & =\rho_{z}^{1 c}, \\
g_{n n}^{1 c} & =\vec{\nabla} \rho_{0}^{1 c} \cdot \vec{\nabla} \rho_{0}^{1 c}, \\
g_{s s}^{1 c} & =\vec{\nabla} \rho_{z}^{1 c} \cdot \vec{\nabla} \rho_{z}^{1 c}, \\
g_{n s}^{1 c} & =\vec{\nabla} \rho_{0}^{1 c} \cdot \vec{\nabla} \rho_{z}^{1 c} .
\end{aligned}
$$

However, parameterizations of the exchange-correlation energy density that depend only on one component of the spin density are inadequate for two- (2c) and fourcomponent (4c) theories, since they yield expressions that lack rotational invariance, and fail to fully capture the SOC effects. For LDA functionals this problem was overcome by the substitution of the z-component of spin density by its magnitude, $\rho_{z} \rightarrow|\vec{\rho}|$, a technique developed by van Wüllen. ${ }^{47}$ This re-parametrization is referred to as the noncollinear ansatz. Unfortunately, the direct extension of this parametrization to GGA functionals, i.e., $\vec{\nabla} \rho_{z} \rightarrow \vec{\nabla}|\vec{\rho}|$, is known to be numerically unstable. ${ }^{31,44}$ A robust solution to this problem was proposed by Scalmani and Frisch, ${ }^{44}$ leading to the following fully rotationally invariant variables ${ }^{53}$

$$
\begin{aligned}
n & =\rho_{0}, \\
s & =\sqrt{\vec{\rho} \cdot \vec{\rho}}, \\
g_{n n} & =\vec{\nabla} \rho_{0} \cdot \vec{\nabla} \rho_{0}, \\
g_{s s} & =\vec{\nabla} \rho_{k} \cdot \vec{\nabla} \rho_{k}, \\
g_{n s} & =f_{\nabla} \sqrt{\vec{g} \cdot \vec{g}}=f_{\nabla} g, \\
g_{k} & =\vec{\nabla} \rho_{0} \cdot \vec{\nabla} \rho_{k}, \\
f_{\nabla} & =\operatorname{sgn}(\vec{g} \cdot \vec{\rho}) .
\end{aligned}
$$

An important distinction between Eqs. (17) and (18) is that in Eq. (17) both $\rho_{z}$ and $\vec{\nabla} \rho_{0} \cdot \vec{\nabla} \rho_{z}$ can have both positive and negative values, while their counterparts, $s$ and $g$ in Eq. (18), are always positive. To resolve this discrepancy a function, $f_{\nabla}$, was introduced in the definition of a noncollinear variable, $g_{n s}$. This solution is made possible by the invariance of the exchange-correlation functionals under interchange of $\alpha$ and $\beta$ variables.

The charge density, $\rho_{0}$, and spin density, $\vec{\rho}$, variables in Eqs. (18) can be obtained using any level of relativistic theory in which the spin-orbit interaction is included variationally. In the present work we consider the Dirac 4c theory

$$
\begin{aligned}
& \rho_{0}=\operatorname{Tr}\left[\left(\mathbf{X}^{\mathrm{RKB}}\right)^{\dagger} \mathbf{X}^{\mathrm{RKB}} \mathbf{D}\right] \\
& \vec{\rho}=\operatorname{Tr}\left[\left(\mathbf{X}^{\mathrm{RKB}}\right)^{\dagger} \overrightarrow{\boldsymbol{\Sigma}} \mathbf{X}^{\mathrm{RKB}} \mathbf{D}\right],
\end{aligned}
$$

where $\overrightarrow{\boldsymbol{\Sigma}}$ is the four-component matrix

$$
\overrightarrow{\boldsymbol{\Sigma}}=\left(\begin{array}{cc}
\vec{\sigma} & 0 \\
0 & \vec{\sigma}
\end{array}\right)
$$

Development of the fully relativistic 4c-LR-KU-TDDFT method described in this article necessitated derivation of new expressions for the noncollinear exchangecorrelation potential, $\mathbf{V}^{\mathrm{xc}}$, and kernel, $\mathbf{K}^{\mathrm{xc}}$, which are applicable to ground states with arbitrary time-reversal symmetry. In contrast to Ref. 35, these expressions do not require calculation of kinetic energy densities $(\tau$ and $\vec{u}$ in Ref. 35), instead making use of the gradient of the overlap distribution matrix, $\vec{\nabla} \boldsymbol{\Omega}$. Consequently, the exchange-correlation potential can be expressed as ${ }^{53}$

$$
\begin{aligned}
V_{\mu \nu}^{\mathrm{xc}}=\frac{\mathrm{d} E^{\mathrm{xc}}}{\mathrm{d} D_{\nu \mu}}=\int & \left(v^{n} \Omega_{\mu \nu}^{0}+v^{s} \frac{\rho_{k}}{s} \Omega_{\mu \nu}^{k}\right. \\
& +v^{g_{n n}} 2 \nabla_{l} \rho_{0} \nabla_{l} \Omega_{\mu \nu}^{0} \\
& +v^{g_{s s}} 2 \nabla_{l} \rho_{k} \nabla_{l} \Omega_{\mu \nu}^{k} \\
& +v^{g_{n s}} f_{\nabla} \frac{g_{k}}{g} \nabla_{l} \rho_{k} \nabla_{l} \Omega_{\mu \nu}^{0} \\
& \left.+v^{g_{n s}} f_{\nabla} \frac{g_{k}}{g} \nabla_{l} \rho_{0} \nabla_{l} \Omega_{\mu \nu}^{k}\right) \mathrm{dV}
\end{aligned}
$$

where auxiliary variables $v^{t}$ and overlap distribution matrices have the form

$$
\begin{gathered}
\Omega_{\mu \nu}^{m}=\frac{\partial \rho_{m}}{\partial D_{\nu \mu}}, \quad \vec{\nabla} \Omega_{\mu \nu}^{m}=\frac{\partial\left(\vec{\nabla} \rho_{m}\right)}{\partial D_{\nu \mu}}, \quad m=0 \ldots 3 \\
v^{t}=\frac{\partial \varepsilon^{\mathrm{xc}}}{\partial t}, \quad t=n, s, g_{n n}, g_{s s}, g_{n s}
\end{gathered}
$$

The exchange--correlation kernel contracted with an arbitrary matrix is written ${ }^{53}$

$$
\begin{aligned}
& K_{\mu \nu, \lambda \tau}^{\mathrm{xc}} \widetilde{D}_{\tau \lambda}=\frac{\mathrm{d} V_{\mu \nu}^{\mathrm{xc}}}{\mathrm{d} D_{\tau \lambda}} \widetilde{D}_{\tau \lambda}= \\
& \int\left\{\left(k^{n n} \tilde{\rho}_{0}+k^{n s} \tilde{s}+k^{n g_{n n}} \tilde{g}_{n n}+k^{n g_{s s}} \tilde{g}_{s s}+k^{n g_{n s}} \tilde{g}_{n s}\right) \Omega_{\mu \nu}^{0}\right. \\
& +\left(k^{s n} \tilde{\rho}_{0}+k^{s s} \tilde{s}+k^{s g_{n n}} \tilde{g}_{n n}+k^{s g_{s s}} \tilde{g}_{s s}+k^{s g_{n s}} \tilde{g}_{n s}\right) \frac{\rho_{k}}{s} \Omega_{\mu \nu}^{k} \\
& +\left(k^{g_{n n} n} \tilde{\rho}_{0}+k^{g_{n n} s} \tilde{s}+k^{g_{n n} g_{n n}} \tilde{g}_{n n}\right. \\
& \left.\quad+k^{g_{n n} g_{s s}} \tilde{g}_{s s}+k^{g_{n n} g_{n s}} \tilde{g}_{n s}\right) 2 \nabla_{l} \rho_{0} \nabla_{l} \Omega_{\mu \nu}^{0} \\
& +\left(k^{g_{s s} n} \tilde{\rho}_{0}+k^{g_{s s} s} \tilde{s}+k^{g_{s s} g_{n n}} \tilde{g}_{n n}\right. \\
& \left.\quad+k^{g_{s s} g_{s s}} \tilde{g}_{s s}+k^{g_{s s} g_{n s}} \tilde{g}_{n s}\right) 2 \nabla_{l} \rho_{k} \nabla_{l} \Omega_{\mu \nu}^{k} \\
& +\left(k^{g_{n s} n} \tilde{\rho}_{0}+k^{g_{n s} s} \tilde{s}+k^{g_{n s} g_{n n}} \tilde{g}_{n n}+k^{g_{n s} g_{s s}} \tilde{g}_{s s}\right. \\
& \left.\quad+k^{g_{n s} g_{n s}} \tilde{g}_{n s}\right) f_{\nabla} \frac{g_{k}}{g}\left(\nabla_{l} \rho_{k} \nabla_{l} \Omega_{\mu \nu}^{0}+\nabla_{l} \rho_{0} \nabla_{l} \Omega_{\mu \nu}^{k}\right) \\
& +\frac{v^{s}}{s}\left(\tilde{\rho}_{k}-\frac{\rho_{k}}{s} \tilde{s}\right) \Omega_{\mu \nu}^{k}+v^{g_{n n}} 2 \nabla_{l} \tilde{\rho}_{0} \nabla_{l} \Omega_{\mu \nu}^{0}+v^{g_{s s}} 2 \nabla_{l} \tilde{\rho}_{k} \nabla_{l} \Omega_{\mu \nu}^{k} \\
& +\frac{v^{g_{n s}}}{g} f_{\nabla}\left(g_{k} \nabla_{l} \tilde{\rho}_{k}+\tilde{g}_{k} \nabla_{l} \rho_{k}-\frac{g_{k} \nabla_{l} \rho_{k}}{g} \tilde{g}\right) \nabla_{l} \Omega_{\mu \nu}^{0} \\
& \left.+\frac{v^{g_{n s}}}{g} f_{\nabla}\left(g_{k} \nabla_{l} \tilde{\rho}_{0}+\tilde{g}_{k} \nabla_{l} \rho_{0}-\frac{g_{k} \nabla_{l} \rho_{0}}{g} \tilde{g}\right) \nabla_{l} \Omega_{\mu \nu}^{k}\right\} \mathrm{dV},
\end{aligned}
$$


with auxiliary variables defined as

$$
\begin{aligned}
\tilde{s} & =\frac{\rho_{k} \tilde{\rho}_{k}}{s}, \\
\tilde{g}_{n n} & =2 \nabla_{l} \rho_{0} \nabla_{l} \tilde{\rho}_{0} \\
\tilde{g}_{s s} & =2 \nabla_{l} \rho_{k} \nabla_{l} \tilde{\rho}_{k}, \\
\tilde{g}_{n s} & =f_{\nabla} \frac{g_{k} \tilde{g}_{k}}{g}=f_{\nabla} \tilde{g} \\
\tilde{g}_{k} & =\nabla_{l} \rho_{k} \nabla_{l} \tilde{\rho}_{0}+\nabla_{l} \tilde{\rho}_{k} \nabla_{l} \rho_{0}, \\
\tilde{\rho}_{m} & =\Omega_{\lambda \tau}^{m} \widetilde{D}_{\tau \lambda}, \quad m=0 \ldots 3, \quad t, u=n, s, g_{n n}, g_{s s}, g_{n s} . \\
k^{t u} & =\frac{\partial \varepsilon^{\mathrm{xc}}}{\partial t \partial u}, \quad t
\end{aligned}
$$

For clarity we keep $k^{t u}$ and $k^{u t}$ separate in expressions for the DFT kernel, despite them being equal, i.e., $k^{t u}=k^{u t}$.

Numerical instabilities referred to in this work affect GGA DFT potentials and kernels for systems with degenerate ground state when rotational invariance of the exchange-correlation energy is introduced via noncollinear ansatz. This holds for all noncollinear ansatzes discussed in this work, since they all incorporate, in one way or another, a square root function. The derivative of a square root function at zero is not defined, which leads to numerical problems in regions where the spin densities or their gradients approach zero. Although the potential and kernel derived from the noncollinear ansatz (18) are not completely free from these problems, they are less prominent than in a potential and kernel developed using the parametrization $\vec{\nabla} \rho_{z} \rightarrow \vec{\nabla}|\vec{\rho}|$. In the latter parametrization, large cutoff thresholds must be applied $^{54}$ if these instabilities are to be avoided. In the following we will address these issues in the framework of the noncollinear ansatz (18), rigorously wherever possible, and via re-definition of the functional where not.

\section{Limit cases: $s=0 \vee g=0$}

It was noted previously that for the choice of noncollinear variables

$$
\begin{gathered}
\rho_{z} \rightarrow s, \\
\vec{\nabla} \rho_{z} \rightarrow \vec{\nabla} s,
\end{gathered}
$$

the resulting exchange--correlation potential and kernel are numerically unstable, ${ }^{31,44}$ and therefore the ansatz (33) must be rejected. We argue that this instability originates in the ill-defined derivative of the variable, $s$, when $s=0$. A manifestation of this ill behavior is the non-existence of the limit

$$
\lim _{s(0) \rightarrow 0} \lim _{\lambda \rightarrow 0} \frac{\rho_{k}(\lambda)}{s(\lambda)} \neq \lim _{\lambda \rightarrow 0} \lim _{s(0) \rightarrow 0} \frac{\rho_{k}(\lambda)}{s(\lambda)},
$$

where $\lambda$ is a perturbation parameter. Issues with such limit cases affect not only the rejected noncollinear ansatz [Eq. (33)], but also the Scalmani and Frisch noncollinear definition [Eq. (18)] for variables $s$ and $g_{n s}$, albeit in a less severe manner. In the case of LDA functionals, which do not require evaluation of spatial gradients of the spin density, the situation can be resolved rigorously. The solution was first described in the context of TDDFT theory by Wang and Ziegler ${ }^{49}$, who observed that

$$
\forall \vec{r} \text { s.t. } s=0: \quad v^{s}=k^{n s}=0 \quad \text { and } \quad \frac{v^{s}}{s}=k^{s s} .
$$

It is possible to generalize the expression, $v^{s}=k^{n s}=0$, in the context of GGA functionals, in which gradients of the spin density must be considered

$$
\begin{array}{cc} 
& \forall \vec{r} \text { s.t. } s=g_{n s}=0: \\
& v^{s}=v^{g_{n s}}=0 \\
\text { and } \quad & k^{n s}=k^{n g_{n s}}=k^{g_{n n} s}= \\
& k^{g_{n n} g_{n s}}=k^{g_{s s} s}=k^{g_{s s} g_{n s}}=0 .
\end{array}
$$

This extension relies on the invariance of exchangecorrelation functionals under interchange of $\alpha$ and $\beta$ variables. Unfortunately, attempts to generalize the last term in expression (35) have proven less successful, yielding the expressions

$$
\begin{gathered}
\qquad \vec{r} \text { s.t. } s=g_{n s}=0: \\
\frac{v^{s}}{s}=k^{s s}+\text { ill-defined } \\
\text { and } \frac{v^{g_{n s}}}{g}=k^{g_{n s} g_{n s}}+\text { ill-defined, }
\end{gathered}
$$

which suffer from the same ill behavior associated with the non-existence of the limit specified in (34).

Nevertheless, two distinct noncollinear DFT kernels have been proposed. One by Bast et al. ${ }^{32}$ in the framework of LR-TDDFT theory, and another by Komorovsky et al. ${ }^{55}$ in the context of calculation of nuclear spinrotation constants. Both kernels were formulated for the closed-shell limit, i.e., when the perturbation-free density matrix is symmetric under time reversal, and thus the perturbation-free spin density and its gradients vanish

$$
\vec{\rho}=0 \wedge \vec{\nabla} \vec{\rho}=0 \quad \Rightarrow \quad s=0 \wedge g_{n s}=0 .
$$

Both methods satisfy two necessary conditions of any noncollinear DFT methodology intended for treatment of systems with a non-degenerate ground state. First, that a form for their exchange-correlation potential is ${ }^{53}$

$$
V_{\mu \nu}^{\mathrm{xc}}=\int\left(v^{n} \Omega_{\mu \nu}^{0}+v^{g_{n n}} 2 \nabla_{l} \rho_{0} \nabla_{l} \Omega_{\mu \nu}^{0}\right) \mathrm{dV},
$$

and second, that their collinear limit

$$
\begin{gathered}
\vec{\rho} \rightarrow \rho_{z}, \\
\vec{\nabla} \vec{\rho} \rightarrow \vec{\nabla} \rho_{z},
\end{gathered}
$$

has the form of standard non-relativistic DFT functionals. The validity of both methods is supported by the 
numerical results in Refs. 32 and 55. Hovewer, in the present work we have found that the extension of the noncollinear kernel in Ref. 55 to the LR-TDDFT domain gives unsatisfactory results when applied to atoms in the $\mathrm{Zn}-\mathrm{Hg}$ series, and that the error in the degeneracy of the energy spectra can be as large as $1 \%$. Due to these observations the present authors suggest avoiding the use of this kernel for calculation of energy spectra and to reconsider its use in the calculation of magnetic properties. We propose the use of the kernel presented in Ref. 32 for case (36), with a slight modification accommodating the non-equivalence (46), detailed below ${ }^{53}$

$$
\begin{gathered}
\forall \vec{r} \text { s.t. } s=g_{n s}=0: \\
V_{\mu \nu}^{\mathrm{xc}}=\int\left(v^{n} \Omega_{\mu \nu}^{0}+v^{g_{n n}} 2 \nabla_{l} \rho_{0} \nabla_{l} \Omega_{\mu \nu}^{0}\right. \\
\left.+v^{g_{s s}} 2 \nabla_{l} \rho_{k} \nabla_{l} \Omega_{\mu \nu}^{k}\right) \mathrm{dV}
\end{gathered}
$$

and

$$
\begin{aligned}
& K_{\mu \nu, \lambda \tau}^{\mathrm{xc}} \widetilde{D}_{\tau \lambda}= \\
& \int\left\{\left(k^{n n} \tilde{\rho}_{0}+k^{n g_{n n}} \tilde{g}_{n n}+k^{n g_{s s}} \tilde{g}_{s s}\right) \Omega_{\mu \nu}^{0}\right. \\
& +\left(k^{g_{n n} n} \tilde{\rho}_{0}+k^{g_{n n} g_{n n}} \tilde{g}_{n n}+k^{g_{n n} g_{s s}} \tilde{g}_{s s}\right) 2 \nabla_{l} \rho_{0} \nabla_{l} \Omega_{\mu \nu}^{0} \\
& +\left(k^{g_{s s} n} \tilde{\rho}_{0}+k^{g_{s s} g_{n n}} \tilde{g}_{n n}+k^{g_{s s} g_{s s}} \tilde{g}_{s s}\right) 2 \nabla_{l} \rho_{k} \nabla_{l} \Omega_{\mu \nu}^{k} \\
& +\left(k^{g_{n s} s} \tilde{\rho}_{k}+k^{g_{n s} g_{n s}} \tilde{g}_{k}\right)\left(\nabla_{l} \rho_{k} \nabla_{l} \Omega_{\mu \nu}^{0}+\nabla_{l} \rho_{0} \nabla_{l} \Omega_{\mu \nu}^{k}\right) \\
& +\left(k^{s s} \tilde{\rho}_{k}+k^{s g_{n s}} \tilde{g}_{k}\right) \Omega_{\mu \nu}^{k} \\
& \left.+v^{g_{n n}} 2 \nabla_{l} \tilde{\rho}_{0} \nabla_{l} \Omega_{\mu \nu}^{0}+v^{g_{s s}} 2 \nabla_{l} \tilde{\rho}_{k} \nabla_{l} \Omega_{\mu \nu}^{k}\right\} \mathrm{dV} .
\end{aligned}
$$

This definition reflects the fact the implication in expression (40) can not be extended to the equivalence

$$
\vec{\rho}=0 \wedge \vec{\nabla} \vec{\rho}=0 \quad \not \quad s=0 \wedge g_{n s}=0 .
$$

From consideration of Eqs. (36)-(39), it is apparent that under condition (43) the Scalmani and Frisch noncollinear ansatz, Eqs. (18), leads to the exchangecorrelation potential as specified in (44), and recovers all terms in the kernel definition in Eq. (45), with the exception of those containing $k^{g_{n s} s}$. These terms are not well defined, due to the ill behavior of the limit (34), therefore, we define them using expressions from Ref. 32 as these provide numerically stable results consistent with experimental data. In summary, the expressions (43)-(45) have the correct collinear limit, are rotationally invariant, and numerically stable. Crucially, they are consistent with the noncollinear ansatz of Scalmani and Frisch in all cases in which the expressions (22) and (25) are well defined in the limit $\left(s \rightarrow 0 \wedge g_{n s} \rightarrow 0\right)$. Furthermore, they are equivalent, in the closed shell limit, to the well behaved kernel of Bast et $a l .^{32}$.

It remains to discuss two mixed cases

$$
\begin{aligned}
& \forall \vec{r} \text { s.t. } s=0 \wedge g_{n s} \neq 0, \\
& \forall \vec{r} \text { s.t. } s \neq 0 \wedge g_{n s}=0 .
\end{aligned}
$$

Unfortunately, both of these cases are more challenging than (36), since Eqs. (37) and (38) are not valid, and the corresponding functions are in general nonzero. Furthermore, the expressions, $\frac{v^{s}}{s}$ and $\frac{v^{g_{n s}}}{g}$, relevant to the calculation of the non-collinear potential and kernel, are divergent for the first [Eq. (47)] and the second case [Eq. (48)], respectively. To overcome this problem the exchange-correlation potential and kernel are defined in the collinear manner when necessary ${ }^{53}$

$$
\begin{aligned}
& \forall \vec{r} \text { s.t. } s=0 \wedge g_{n s} \neq 0: \\
& s=\rho_{\gamma}, \quad f_{\nabla}=\operatorname{sgn}\left(g_{\gamma}\right) \\
& V_{\mu \nu}^{\mathrm{xc}}(\gamma)=\int\left(v^{n} \Omega_{\mu \nu}^{0}+v^{s} \Omega_{\mu \nu}^{\gamma}\right. \\
&+v^{g_{n n}} 2 \nabla_{l} \rho_{0} \nabla_{l} \Omega_{\mu \nu}^{0}+v^{g_{s s}} 2 \nabla_{l} \rho_{k} \nabla_{l} \Omega_{\mu \nu}^{k} \\
&+v^{g_{n s}} f_{\nabla} \frac{g_{k}}{g} \nabla_{l} \rho_{k} \nabla_{l} \Omega_{\mu \nu}^{0} \\
&\left.+v^{g_{n s}} f_{\nabla} \frac{g_{k}}{g} \nabla_{l} \rho_{0} \nabla_{l} \Omega_{\mu \nu}^{k}\right) \mathrm{dV}
\end{aligned}
$$

and

$$
\begin{aligned}
& K_{\mu \nu, \lambda \tau}^{\mathrm{xc}}(\gamma) \widetilde{D}_{\tau \lambda}=\int\{ \\
& \left(k^{n n} \tilde{\rho}_{0}+k^{n s} \tilde{\rho}_{\gamma}+k^{n g_{n n}} \tilde{g}_{n n}+k^{n g_{s s}} \tilde{g}_{s s}+k^{n g_{n s}} \tilde{g}_{n s}\right) \Omega_{\mu \nu}^{0} \\
& +\left(k^{s n} \tilde{\rho}_{0}+k^{s s} \tilde{\rho}_{\gamma}+k^{s g_{n n}} \tilde{g}_{n n}+k^{s g_{s s}} \tilde{g}_{s s}+k^{s g_{n s}} \tilde{g}_{n s}\right) \Omega_{\mu \nu}^{\gamma} \\
& +\left(k^{g_{n n} n} \tilde{\rho}_{0}+k^{g_{n n} s} \tilde{\rho}_{\gamma}+k^{g_{n n} g_{n n}} \tilde{g}_{n n}\right. \\
& \left.+k^{g_{n n} g_{s s}} \tilde{g}_{s s}+k^{g_{n n} g_{n s}} \tilde{g}_{n s}\right) 2 \nabla_{l} \rho_{0} \nabla_{l} \Omega_{\mu \nu}^{0} \\
& +\left(k^{g_{s s} n} \tilde{\rho}_{0}+k^{g_{s s} s} \tilde{\rho}_{\gamma}+k^{g_{s s} g_{n n}} \tilde{g}_{n n}\right. \\
& \left.+k^{g_{s s} g_{s s}} \tilde{g}_{s s}+k^{g_{s s} g_{n s}} \tilde{g}_{n s}\right) 2 \nabla_{l} \rho_{k} \nabla_{l} \Omega_{\mu \nu}^{k} \\
& +\left(k^{g_{n s} n} \tilde{\rho}_{0}+k^{g_{n s} s} \tilde{\rho}_{\gamma}+k^{g_{n s} g_{n n}} \tilde{g}_{n n}+k^{g_{n s} g_{s s}} \tilde{g}_{s s}\right. \\
& \left.+k^{g_{n s} g_{n s}} \tilde{g}_{n s}\right) f_{\nabla} \frac{g_{k}}{g}\left(\nabla_{l} \rho_{k} \nabla_{l} \Omega_{\mu \nu}^{0}+\nabla_{l} \rho_{0} \nabla_{l} \Omega_{\mu \nu}^{k}\right) \\
& +v^{g_{n n}} 2 \nabla_{l} \tilde{\rho}_{0} \nabla_{l} \Omega_{\mu \nu}^{0}+v^{g_{s s}} 2 \nabla_{l} \tilde{\rho}_{k} \nabla_{l} \Omega_{\mu \nu}^{k} \\
& +\frac{v^{g_{n s}}}{g} f_{\nabla}\left(g_{k} \nabla_{l} \tilde{\rho}_{k}+\tilde{g}_{k} \nabla_{l} \rho_{k}-\frac{g_{k} \nabla_{l} \rho_{k}}{g} \tilde{g}\right) \nabla_{l} \Omega_{\mu \nu}^{0} \\
& \left.+\frac{v^{g_{n s}}}{g} f_{\nabla}\left(g_{k} \nabla_{l} \tilde{\rho}_{0}+\tilde{g}_{k} \nabla_{l} \rho_{0}-\frac{g_{k} \nabla_{l} \rho_{0}}{g} \tilde{g}\right) \nabla_{l} \Omega_{\mu \nu}^{k}\right\} \mathrm{dV} . \\
& \forall \vec{r} \text { s.t. } s \neq 0 \wedge g_{n s}=0 \text { : } \\
& g_{n s}=\vec{\nabla} \rho_{0} \cdot \vec{\nabla} \rho_{\gamma}, \\
& V_{\mu \nu}^{\mathrm{xc}}(\gamma)=\int\left(v^{n} \Omega_{\mu \nu}^{0}+v^{s} \frac{\rho_{k}}{s} \Omega_{\mu \nu}^{k}\right. \\
& +v^{g_{n n}} 2 \nabla_{l} \rho_{0} \nabla_{l} \Omega_{\mu \nu}^{0}+v^{g_{s s}} 2 \nabla_{l} \rho_{k} \nabla_{l} \Omega_{\mu \nu}^{k} \\
& \left.+v^{g_{n s}} \nabla_{l} \rho_{\gamma} \nabla_{l} \Omega_{\mu \nu}^{0}+v^{g_{n s}} \nabla_{l} \rho_{0} \nabla_{l} \Omega_{\mu \nu}^{\gamma}\right) \mathrm{dV},
\end{aligned}
$$

and 
TABLE I. Summary of the expressions for noncollinear exchange-correlation potential and kernel. ${ }^{a}$

\begin{tabular}{ccccc}
\hline \hline case & $\forall \vec{r}$ s.t. & variables $^{\mathrm{b}}$ & $\mathbf{V}^{\mathrm{xc}}$ & $\mathbf{K}^{\mathrm{xc}}$ \\
\hline 1. & $s>\Theta \wedge g_{n s}>\Theta$ & & $(22)$ & $(25)$ \\
2. & $s>\Theta \wedge g_{n s} \leq \Theta$ & $(54)$ & $(55)$ & $(56)$ \\
3. & $s \leq \Theta \wedge g_{n s}>\Theta$ & $(50)$ & $(51)$ & $(52)$ \\
4. & $s \leq \Theta \wedge g_{n s} \leq \Theta$ & $s=0 \quad g_{n s}=0$ & $(44)$ & $(45)$ \\
\hline \hline
\end{tabular}

a Numbers in the table represent equation numbers.

b The final variable set is obtained after the data from the table is substituted to Eq. (18).

$K_{\mu \nu, \lambda \tau}^{\mathrm{xc}}(\gamma) \widetilde{D}_{\tau \lambda}=$

$\int\left\{\left(k^{n n} \tilde{\rho}_{0}+k^{n s} \tilde{s}+k^{n g_{n n}} \tilde{g}_{n n}+k^{n g_{s s}} \tilde{g}_{s s}+k^{n g_{n s}} \tilde{g}_{\gamma}\right) \Omega_{\mu \nu}^{0}\right.$

$+\left(k^{s n} \tilde{\rho}_{0}+k^{s s} \tilde{s}+k^{s g_{n n}} \tilde{g}_{n n}+k^{s g_{s s}} \tilde{g}_{s s}+k^{s g_{n s}} \tilde{g}_{\gamma}\right) \frac{\rho_{k}}{s} \Omega_{\mu \nu}^{k}$

$+\left(k^{g_{n n} n} \tilde{\rho}_{0}+k^{g_{n n} s} \tilde{s}+k^{g_{n n} g_{n n}} \tilde{g}_{n n}\right.$

$\left.+k^{g_{n n} g_{s s}} \tilde{g}_{s s}+k^{g_{n n} g_{n s}} \tilde{g}_{\gamma}\right) 2 \nabla_{l} \rho_{0} \nabla_{l} \Omega_{\mu \nu}^{0}$

$+\left(k^{g_{s s} n} \tilde{\rho}_{0}+k^{g_{s s} s} \tilde{s}+k^{g_{s s} g_{n n}} \tilde{g}_{n n}\right.$

$\left.+k^{g_{s s} g_{s s}} \tilde{g}_{s s}+k^{g_{s s} g_{n s}} \tilde{g}_{\gamma}\right) 2 \nabla_{l} \rho_{k} \nabla_{l} \Omega_{\mu \nu}^{k}$

$+\left(k^{g_{n s} n} \tilde{\rho}_{0}+k^{g_{n s} s} \tilde{s}+k^{g_{n s} g_{n n}} \tilde{g}_{n n}+k^{g_{n s} g_{s s}} \tilde{g}_{s s}\right.$

$\left.+k^{g_{n s} g_{n s}} \tilde{g}_{\gamma}\right)\left(\nabla_{l} \rho_{\gamma} \nabla_{l} \Omega_{\mu \nu}^{0}+\nabla_{l} \rho_{0} \nabla_{l} \Omega_{\mu \nu}^{\gamma}\right)$

$+\frac{v^{s}}{s}\left(\tilde{\rho}_{k}-\frac{\rho_{k}}{s} \tilde{s}\right) \Omega_{\mu \nu}^{k}+v^{g_{n n}} 2 \nabla_{l} \tilde{\rho}_{0} \nabla_{l} \Omega_{\mu \nu}^{0}$

$\left.+v^{g_{s s}} 2 \nabla_{l} \tilde{\rho}_{k} \nabla_{l} \Omega_{\mu \nu}^{k}+v^{g_{n s}}\left(\nabla_{l} \tilde{\rho}_{\gamma} \nabla_{l} \Omega_{\mu \nu}^{0}+\nabla_{l} \tilde{\rho}_{0} \nabla_{l} \Omega_{\mu \nu}^{\gamma}\right)\right\} \mathrm{dV}$.

Evaluation of derivatives of the exchange-correlation energy density, $\varepsilon^{\mathrm{xc}}$, in the above expressions make use of the definitions of noncollinear variables in (18), with the exception of those variables defined in Eqs. (50) and (54). The free index, $\gamma$, represents the collinear direction obtained as the index of the maximum element in the absolute value of the vector $\int \vec{\rho} \mathrm{dV}$ and $\int \vec{g} \mathrm{dV}$ in the case of Eqs. (49)-(52) and (53)-(56), respectively. The proposed expressions (49)-(56) satisfy the collinear limit, are numerically stable, but are not fully rotationally invariant.

In practical calculations comparison to a numerical threshold, $\Theta$, is used to determine which of the cases, (43), (49), and (53), is relevant, and which set of expressions should be applied. For convenience, the definition of the proposed DFT potential and kernel is summarized in Table I.

Remarks:

- The regularization threshold, $\Theta$, in Table I is part of the definition of the noncollinear potential and kernel.

- The exchange-correlation potential and kernel in Table I has the proper collinear limit (42), and the closed-shell limit of well behaved kernel of Bast et $a l .{ }^{32}$. Rotational invariance is broken in some terms for the second and third case.
- In Ref. $35 \mathrm{UO}_{2}^{2+}$ is found to exhibit non-zero magnetization. However, this system is typically thought to have a non-degenerate ground state with a single-determinant Kramers-restricted configuration $^{32,36-39}$, which is in contradiction to findings in Ref. 35, since non-degenerate states are nonmagnetic. One possible explanation is that the noncollinear DFT kernel used in Ref. 35 based on the Scalmani and Frisch ansatz ${ }^{44}$ does not satisfy the collinear limit. Using the noncollinear DFT potential defined in Table I we obtain a Kramersrestricted wave function when starting from the one-component closed-shell singlet Slater determinant. When starting from a high spin triplet Slater determinant the SCF procedure converges to a Kramers-unrestricted wave function, however, it has a higher energy than the restricted one. This result is consistent with the literature, in which the ground state of $\mathrm{UO}_{2}^{2+}$ system is considered nondegenerate and thus non-magnetic.

- The sign function in the definition (18) has a discontinuity at zero. This leads to additional expressions involving the Dirac delta function. For example, the exchange-correlation potential (22) should have an additional term ${ }^{53}$

$$
\begin{gathered}
2 \int v^{g_{n s}} g \delta(\vec{\rho} \cdot \vec{g})\left(g_{k} \Omega_{\mu \nu}^{k}+\rho_{k} \nabla_{l} \rho_{k} \nabla_{l} \Omega_{\mu \nu}^{0}\right. \\
\left.+\rho_{k} \nabla_{l} \rho_{0} \nabla_{l} \Omega_{\mu \nu}^{k}\right) \mathrm{dV} .
\end{gathered}
$$

This issue is partially solved by redefinition of the variable $g_{n s}$ in cases 2-4 in Table I. The remaining, unlikely case that vectors $\vec{\rho}$ and $\vec{g}$ are non-zero and perpendicular is discarded. Similar issues involving the sign function, $\operatorname{sgn}\left(g_{\gamma}\right)$, in the third case in Table I are also dealt with by discarding the relevant terms.

- The noncollinear GGA exchange-correlation potential and kernel, Table I, are formulated for the general matrix $\widetilde{\mathbf{D}}$, hence these expressions can be easily rewritten to take advantage of any combination of Hermitian and time-reversal symmetry possessed by the matrix $\widetilde{\mathbf{D}}$. For example, Hermitian symmetry and time-reversal antisymmetry can be utilized in relativistic methods for the calculation of NMR shielding or indirect spin-spin coupling constants.

- One of the disadvantages of the Scalmani and Frisch noncollinear ansatz $z^{44}$ is the equal treatment of transverse and longitudinal gradients of the spin density. In the work of Eich et al. ${ }^{41}$ it was demonstrated that in the case of spin-polarized electron gas the transverse and longitudinal gradients have different dependance on the spin polarization, $\frac{s}{n}$, and that the Scalmani and Frisch non- 
collinear ansatz is only justified either in weak spinpolarization limit, or when the system is strongly inhomogeneous (a relatively large transverse component of the spin density gradient). The systems studied in this work fall within the weak spin polarization regime. For example, the spin polarization on the $\mathrm{Pa}-\mathrm{Cl}$ bond does not exceed 0.01. This is consistent with the observation that the spin density is largely due to a single unpaired electron localized around the $\mathrm{Pa}$ atom, where the charge density is significantly larger than the spin-density.

\section{Eigenproblem solver (EPS)}

Taking a direct approach to the solution of Eq. (3) is not feasible due to the large dimension of the matrix. Consequently, iterative algorithms that only require calculation of the matrix-vector product are utilized. A further complication is that the eigenproblem (3) is defined over the field of complex numbers and the corresponding matrix is non-Hermitian. In this work we use a modified version the Davidson algorithm, ${ }^{56}$ which incorporates the preconditioning described in Ref. 57, and which adds four trial vectors to the subspace at each iteration instead of only one.

The paired structure of the Eq. (3) was recognized first by Olsen et al. ${ }^{58}$ who noted that for each solution with eigenvalue $\omega$ there exists a solution with eigenvalue $-\omega$, i.e.,

$$
\begin{aligned}
\left(\begin{array}{cc}
\mathbf{A} & \mathbf{B} \\
-\mathbf{B}^{*} & -\mathbf{A}^{*}
\end{array}\right)\left(\begin{array}{l}
\mathbf{X} \\
\mathbf{Y}
\end{array}\right) & =\omega\left(\begin{array}{l}
\mathbf{X} \\
\mathbf{Y}
\end{array}\right) \\
\Rightarrow \quad\left(\begin{array}{cc}
\mathbf{A} & \mathbf{B} \\
-\mathbf{B}^{*} & -\mathbf{A}^{*}
\end{array}\right)\left(\begin{array}{l}
\mathbf{Y}^{*} \\
\mathbf{X}^{*}
\end{array}\right) & =-\omega\left(\begin{array}{l}
\mathbf{Y}^{*} \\
\mathbf{X}^{*}
\end{array}\right) .
\end{aligned}
$$

In the version of the Davidson-Olsen algorithm proposed in the present article a pair of left eigenvectors of Eq. (3) are added in addition to the pair in Eq. (58). Due to the structure of the $\mathbf{A}$ and $\mathbf{B}$ matrices

$$
\begin{aligned}
\mathbf{A}^{\dagger} & =\mathbf{A}, \\
\mathbf{B}^{\top} & =\mathbf{B},
\end{aligned}
$$

for the left eigenvector it holds that

$$
\begin{aligned}
\left(\begin{array}{cc}
\mathbf{A} & \mathbf{B} \\
-\mathbf{B}^{*} & -\mathbf{A}^{*}
\end{array}\right)\left(\begin{array}{c}
\mathbf{X} \\
\mathbf{Y}
\end{array}\right) & =\omega\left(\begin{array}{c}
\mathbf{X} \\
\mathbf{Y}
\end{array}\right) \\
\Rightarrow \quad\left(\begin{array}{cc}
\mathbf{A} & \mathbf{B} \\
-\mathbf{B}^{*} & -\mathbf{A}^{*}
\end{array}\right)^{\top}\left(\begin{array}{c}
\mathbf{X} \\
-\mathbf{Y}
\end{array}\right) & =\omega\left(\begin{array}{c}
\mathbf{X} \\
-\mathbf{Y}
\end{array}\right) .
\end{aligned}
$$

Accordingly, in each iteration, after a new trial vector is determined, three more trial vectors are added

$$
\left(\begin{array}{c}
\mathbf{X} \\
\mathbf{Y}
\end{array}\right) \rightarrow\left(\begin{array}{c}
\mathbf{X} \\
-\mathbf{Y}
\end{array}\right), \quad\left(\begin{array}{c}
\mathbf{Y}^{*} \\
\mathbf{X}^{*}
\end{array}\right), \quad\left(\begin{array}{c}
\mathbf{Y}^{*} \\
-\mathbf{X}^{*}
\end{array}\right)
$$

To increase the flexibility of the subspace the same basis of trial vectors is used for both sides of the eigenvalue problem. Projection to the subspace of the four new trial vectors requires only two matrix-vector multiplications, one for the left and one for the right trial vector, as the remaining two matrix-vector operations for paired trial eigenvectors, Eq. (58), are determined using the following implication

$$
\begin{aligned}
\left(\begin{array}{cc}
\mathbf{A} & \mathbf{B} \\
-\mathbf{B}^{*} & -\mathbf{A}^{*}
\end{array}\right)\left(\begin{array}{c}
\mathbf{X} \\
\mathbf{Y}
\end{array}\right) & =\left(\begin{array}{c}
\widetilde{\mathbf{X}} \\
\tilde{\mathbf{Y}}
\end{array}\right) \\
\Rightarrow \quad\left(\begin{array}{cc}
\mathbf{A} & \mathbf{B} \\
-\mathbf{B}^{*} & -\mathbf{A}^{*}
\end{array}\right)\left(\begin{array}{c}
\mathbf{Y}^{*} \\
\mathbf{X}^{*}
\end{array}\right) & =\left(\begin{array}{c}
-\widetilde{\mathbf{Y}}^{*} \\
-\widetilde{\mathbf{X}}^{*}
\end{array}\right) .
\end{aligned}
$$

The modification to the Davidson-Olsen algorithm presented above was found to be crucial in ensuring robust convergence of the algorithm when applied to the solution of the 4c-LR-KU-TDDFT equation (3). In the case of Kramers-unrestricted reference states, the modification was found to be necessary to obtain any sensible results at all. For the Kramers-restricted case the improvement was less pronounced, and only marginal for the calculation of the lowest few eigenstates. However, the modification greatly improved performance in cases requiring calculation of a large number of eigenvalues. For example, smooth convergence was observed in calculations of the first 100 eigenvalues of a $\mathrm{Cu}$ atom. When using the PBE0 functional this calculation required 618 matrix vector operations.

\section{COMPUTATIONAL DETAILS}

Unless stated otherwise, all calculations were performed using the developer's version of the RESPECT program. ${ }^{61}$ The molecular geometries of the octahedral systems, $\mathrm{UCl}_{6}^{-}(\mathrm{U}-\mathrm{Cl}=2.5 \AA)$ and $\mathrm{PaCl}_{6}^{2-}(\mathrm{Pa}-\mathrm{Cl}=2.64 \AA)$, have been taken from Ref. 62 , where the distances have been optimized at the spin-free CASPT2 level of theory. In the four-component Dirac-Kohn-Sham calculations the non-relativistic DFT functionals SVWN5, ${ }^{63}$ BLYP ${ }^{64,65}$ B3LYP, ${ }^{63-66} \mathrm{PBE},{ }^{67,68}$ and PBE0 ${ }^{67-69}$ have been employed. The parameter involved in the definition of the noncollinear DFT potential and kernel (see Table I) was set to $\Theta=10^{-16}$. The exchange-correlation nonrelativistic potential and kernel contributions [Eqs. (24) and (32)] were calculated analytically using the automatic differentiation technique implemented in the XCFun library. ${ }^{70}$ Dyall's uncontracted core-valence triple- $\zeta$ basis was used for all atoms. ${ }^{71-76}$ The molecular grid used for integration of the exchange-correlation potential and kernel has an adaptive number of angular grid points and fixed number of radial grid points calculated as follows: Calculations of the reference wave function and excitation energies used $40+n * 10$ and $60+n * 10$ radial grid points, respectively, where $n$ stands for the element's period. The convergence threshold for the residuum in the modified Davidson-Olsen algorithm was set to $10^{-4} \mathrm{au}$. 
TABLE II. Excited state ZFS energies of Group 3 ions $\mathrm{Sc}^{3+}-\mathrm{Ac}^{3+}$ (in meV).

\begin{tabular}{|c|c|c|c|c|c|c|c|c|c|}
\hline & ZFS & SVWN5 ${ }^{\mathrm{a}}$ & $\mathrm{PBE}^{\mathrm{a}}$ & $\mathrm{PBE}^{\mathrm{a}}$ & BLYP $^{\mathrm{a}}$ & B3LYP $^{\mathrm{a}}$ & $\mathrm{DHF}^{\mathrm{a}}$ & $\mathrm{HF}-\mathrm{X} 2 \mathrm{C}^{\mathrm{b}}$ & $\exp ^{c}$ \\
\hline \multirow[t]{6}{*}{$\mathrm{Sc}^{3+}$} & ${ }^{3} P_{1}-{ }^{3} P_{0}$ & 93 & 93 & 93 & 94 & 94 & 92 & - & 84 \\
\hline & ${ }^{3} P_{2}-{ }^{3} P_{1}$ & 189 & 190 & 190 & 191 & 191 & 186 & - & 175 \\
\hline & ${ }^{3} F_{3}-{ }^{3} F_{4}$ & 173 & 181 & 183 & 182 & 183 & 190 & - & 174 \\
\hline & ${ }^{3} F_{2}-{ }^{3} F_{3}$ & 163 & 167 & 167 & 170 & 169 & 166 & - & 161 \\
\hline & $\mathrm{MAE}^{\mathrm{d}}$ & 6 & 9 & 10 & 11 & 11 & 10 & - & - \\
\hline & $\operatorname{MRE}(\%)^{\mathrm{e}}$ & 5.1 & 6.8 & 7.0 & 7.8 & 7.8 & 7.0 & - & - \\
\hline \multirow[t]{6}{*}{$\mathrm{Y}^{3+}$} & ${ }^{3} P_{1}-{ }^{3} P_{0}$ & 196 & 209 & 216 & 205 & 210 & 230 & 228 & 213 \\
\hline & ${ }^{3} P_{2}-{ }^{3} P_{1}$ & 382 & 415 & 432 & 404 & 416 & 469 & 464 & 430 \\
\hline & ${ }^{3} F_{3}-{ }^{3} F_{4}$ & 221 & 285 & 306 & 284 & 294 & 375 & 381 & 281 \\
\hline & ${ }^{3} F_{2}-{ }^{3} F_{3}$ & 251 & 376 & 401 & 369 & 381 & 468 & 468 & 376 \\
\hline & $\mathrm{MAE}^{\mathrm{d}}$ & 62 & 6 & 14 & 11 & 9 & 60 & 60 & - \\
\hline & $\operatorname{MRE}(\%)^{\mathrm{e}}$ & 18.4 & 1.7 & 4.4 & 3.2 & 2.7 & 18.7 & 18.8 & - \\
\hline \multirow[t]{6}{*}{$\mathrm{La}^{3+}$} & ${ }^{3} P_{1}-{ }^{3} P_{0}$ & 244 & 275 & 290 & 261 & 272 & 329 & 328 & 287 \\
\hline & ${ }^{3} P_{2}-{ }^{3} P_{1}$ & 450 & 518 & 555 & 484 & 511 & 651 & 651 & 552 \\
\hline & ${ }^{3} F_{3}-{ }^{3} F_{4}$ & 81 & 176 & 217 & 171 & 191 & 373 & 372 & 171 \\
\hline & ${ }^{3} F_{2}-{ }^{3} F_{3}$ & 236 & 395 & 441 & 368 & 387 & 627 & 642 & 353 \\
\hline & $\mathrm{MAE}^{\mathrm{d}}$ & 88 & 23 & 35 & 27 & 28 & 154 & 158 & - \\
\hline & $\operatorname{MRE}(\%)^{\mathrm{e}}$ & 29.8 & 6.3 & 13.4 & 6.4 & 8.5 & 57.1 & 57.9 & - \\
\hline \multirow[t]{4}{*}{$\mathrm{Ac}^{3+}$} & ${ }^{3} P_{1}-{ }^{3} P_{0}$ & 304 & 356 & 381 & 324 & 341 & 449 & - & - \\
\hline & ${ }^{3} P_{2}-{ }^{3} P_{1}$ & 454 & 558 & 627 & 479 & 534 & 775 & - & - \\
\hline & ${ }^{3} F_{3}-{ }^{3} F_{4}$ & 308 & 210 & 134 & 196 & 155 & 191 & - & - \\
\hline & ${ }^{3} F_{2}-{ }^{3} F_{3}$ & 457 & 563 & 568 & 517 & 513 & 669 & - & - \\
\hline
\end{tabular}

a Results obtained using the 4c-LR-KU-TDDFT method developed in this work.

b Data taken from Ref. 59.

c Data taken from NISC Atomic Spectra Database. ${ }^{60}$

d Mean absolute error.

e Mean relative error.

\section{RESULTS AND DISCUSSION}

Atomic systems with a non-degenerate ground state: To demonstrate the performance of the noncollinear DFT kernel of Bast et al. ${ }^{32}$ and the robustness of the presented eigenvalue solver we examined excited state zero-field splittings of Group 3 elements $\mathrm{Sc}^{3+}-\mathrm{Ac}^{3+}$ (Table II). A Kramers-restricted Slater determinant is sufficient for description of the non-degenerate ground state of these systems.

In all calculations we experienced smooth convergence for all 30 roots, which corresponds to a total of 200 to 400 contractions of the TDDFT kernel with a trial vector. The energetic degeneracy of all calculated roots was reproduced with a precision of $10^{-5 \%}$, while the convergence threshold for the residuum was set to $10^{-4} \mathrm{au}$.

$\mathrm{Y}^{3+}$ and $\mathrm{La}^{3+}$ have also been studied previously at the two-component Hartree-Fock level of theory (denoted HF-X2C in Table II) by Egidi et al. ${ }^{59}$ In Ref. 59 the neglected two-electron spin-orbit effects have been partially corrected by introducing a scaling factor for the one-electron spin-orbit integrals. ${ }^{77,78}$ It was suggested that this approximation is responsible for the discrepancy in the ZFS of states with high angular moment. The largest error can be seen in the ${ }^{3} F_{2}-{ }^{3} F_{3}$ ZFS of $\mathrm{La}^{3+}$ where the calculated value overestimates the experimental result by almost a factor of two. Our calculations show that this is not correct. The agreement between Dirac-Hartree-Fock (DHF) and HF-X2C results is excellent for all splittings, with the exception of the ${ }^{3} F_{2}-{ }^{3} F_{3}$ ZFS of $\mathrm{La}^{3+}$, where a small discrepancy of approximately $2 \%$ was found. Since our four-component results include two-electron spin-own-orbit effects we can conclude that scaling one-electron spin-orbit works well for the systems studied here. The discrepancy in the ZFS of states with high angular moment can be attributed to the missing electron correlation in the DHF and HF-X2C calculations, since the use of GGA DFT functionals consider- 
TABLE III. Low-lying excited states of $\mathrm{Cu}$ (in $\mathrm{eV}$ ).

\begin{tabular}{|c|c|c|c|c|c|c|}
\hline \multirow[b]{2}{*}{${ }^{2} D\left[3 d^{9} 4 s^{2}\right]$} & \multicolumn{3}{|c|}{ 4c-LR-KU-TDDFT ${ }^{\mathrm{a}}$} & \multicolumn{3}{|c|}{ sf-X2C-S-TDA-SOC ${ }^{b}$} \\
\hline & $J_{5 / 2}$ & $J_{3 / 2}$ & $\triangle^{\mathrm{c}}$ & $J_{5 / 2}$ & $J_{3 / 2}$ & $\triangle^{\mathrm{c}}$ \\
\hline DHF & $3.28(0.145)$ & $3.77(0.070)$ & 0.49 & 3.57 & 3.80 & 0.23 \\
\hline SVWN5 & $0.55(0.013)$ & $0.83(0.010)$ & 0.28 & 0.69 & 0.92 & 0.24 \\
\hline BLYP & $0.29(0.034)$ & $0.57(0.022)$ & 0.28 & 0.47 & 0.71 & 0.23 \\
\hline B3LYP & $0.73(0.125)$ & $1.14(0.060)$ & 0.41 & 1.05 & 1.29 & 0.23 \\
\hline PBE & $0.53(0.005)$ & $0.80(0.004)$ & 0.27 & - & - & - \\
\hline PBE0 & $1.32(0.008)$ & $1.61(0.006)$ & 0.29 & - & - & - \\
\hline BHandHLYP & - & - & - & 1.89 & 2.13 & 0.23 \\
\hline $\operatorname{Exp}^{\mathrm{d}}$ & 1.39 & 1.64 & 0.25 & 1.39 & 1.64 & 0.25 \\
\hline
\end{tabular}

a Results obtained using the 4c-LR-KU-TDDFT method developed in this work. Data in the table represent mean values for each degenerate multiplet with the standard deviation in parenthesis.

b Data taken from Ref. 34 .

c $\triangle=J_{3 / 2}-J_{5 / 2}$ represents splitting of ${ }^{2} D\left[3 d^{9} 4 s^{2}\right]$ state.

d Data taken from NISC Atomic Spectra Database. ${ }^{60}$

TABLE IV. Low-lying excited states of $\mathrm{Ag}$ (in $\mathrm{eV}$ ).

\begin{tabular}{|c|c|c|c|c|c|c|}
\hline \multirow[b]{2}{*}{${ }^{2} P\left[4 d^{10} 5 p\right]$} & \multicolumn{3}{|c|}{ 4c-LR-KU-TDDFT ${ }^{\mathrm{a}}$} & \multicolumn{3}{|c|}{ sf-X2C-S-TDA-SOC ${ }^{b}$} \\
\hline & $J_{1 / 2}$ & $J_{3 / 2}$ & $\Delta^{c}$ & $J_{1 / 2}$ & $J_{3 / 2}$ & $\triangle^{\mathrm{c}}$ \\
\hline DHF & $2.82(0.022)$ & $3.05(0.113)$ & 0.23 & 3.15 & 3.27 & 0.12 \\
\hline SVWN5 & $3.88(0.027)$ & $4.06(0.058)$ & 0.18 & 4.32 & 4.47 & 0.14 \\
\hline BLYP & $2.15(2.511)$ & $2.18(2.071)$ & 0.03 & 4.30 & 4.44 & 0.14 \\
\hline B3LYP & $2.68(1.579)$ & $2.71(1.313)$ & 0.03 & 4.13 & 4.27 & 0.14 \\
\hline PBE & $3.64(0.024)$ & $3.83(0.084)$ & 0.19 & - & - & - \\
\hline PBE0 & $3.56(0.016)$ & $3.68(0.031)$ & 0.12 & - & - & - \\
\hline BHandHLYP & - & - & - & 3.87 & 4.01 & 0.14 \\
\hline \multirow[t]{2}{*}{$\operatorname{Exp}^{\mathrm{d}}$} & 3.66 & 3.78 & 0.11 & 3.66 & 3.78 & 0.11 \\
\hline & \multicolumn{3}{|c|}{ 4c-LR-KU-TDDFT ${ }^{\mathrm{a}}$} & \multicolumn{3}{|c|}{ sf-X2C-S-TDA-SOC ${ }^{b}$} \\
\hline${ }^{2} D\left[4 d^{9} 5 s^{2}\right]$ & $J_{5 / 2}$ & $J_{3 / 2}$ & $\triangle^{\mathrm{e}}$ & $J_{5 / 2}$ & $J_{3 / 2}$ & $\triangle^{\mathrm{e}}$ \\
\hline DHF & $4.94(0.094)$ & $5.61(0.061)$ & 0.67 & 5.09 & 5.29 & 0.20 \\
\hline SVWN5 & $2.96(0.018)$ & $3.52(0.013)$ & 0.56 & 3.00 & 3.52 & 0.52 \\
\hline BLYP & $2.76(0.039)$ & $3.33(0.026)$ & 0.57 & 2.84 & 3.36 & 0.52 \\
\hline B3LYP & $3.17(0.070)$ & $3.76(0.040)$ & 0.59 & 3.30 & 3.82 & 0.52 \\
\hline PBE & $2.99(0.042)$ & $3.56(0.029)$ & 0.57 & - & - & - \\
\hline PBE0 & $3.49(0.093)$ & $4.10(0.061)$ & 0.61 & - & - & - \\
\hline BHandHLYP & - & - & - & 3.93 & 4.44 & 0.51 \\
\hline $\operatorname{Exp}^{d}$ & 3.75 & 4.30 & 0.55 & 3.75 & 4.30 & 0.55 \\
\hline
\end{tabular}

a Results obtained using the 4c-LR-KU-TDDFT method developed in this work. Data in the table represent mean values for each degenerate multiplet with the standard deviation in parenthesis.

b Data taken from Ref. 34

c $\triangle=J_{3 / 2}-J_{1 / 2}$ represents splitting of ${ }^{2} P\left[4 d^{10} 5 p\right]$ state.

d Data taken from NISC Atomic Spectra Database. ${ }^{60}$

e $\triangle=J_{3 / 2}-J_{5 / 2}$ represents splitting of ${ }^{2} D\left[4 d^{9} 5 s^{2}\right]$ state.

ably improves both the mean absolute error (MAE) and the mean relative error (MRE) of the calculated results.

Overall, the performance of the GGA DFT functionals is acceptable, with results obtained using hybrid functionals showing little to no improvement over those obtained using pure DFT functionals. As already observed in Ref. 59, Hartree-Fock works well for light elements $\left(\mathrm{Sc}^{3+}\right.$ in this study), but breaks down for heavier ones. Note that the order of energy levels is not always predicted correctly. In the case of $\mathrm{Sc}^{3+}$ calculations, no discrepancies have been found. On the other hand, for $\mathrm{Y}^{3+}$ the energies of the ${ }^{3} P_{2}$ and ${ }^{3} F_{4}$ levels are swapped 
TABLE V. Low-lying excited states of $\mathrm{Au}(\mathrm{in} \mathrm{eV})$

\begin{tabular}{|c|c|c|c|c|c|c|}
\hline \multirow[b]{2}{*}{${ }^{2} D\left[5 d^{9} 6 s^{2}\right]$} & \multicolumn{3}{|c|}{ 4c-LR-KU-TDDFT ${ }^{\mathrm{a}}$} & \multicolumn{3}{|c|}{ sf-X2C-S-TDA-SOC ${ }^{b}$} \\
\hline & $J_{5 / 2}$ & $J_{3 / 2}$ & $\triangle^{c}$ & $J_{5 / 2}$ & $J_{3 / 2}$ & $\triangle^{\mathrm{c}}$ \\
\hline $\mathrm{DHF}$ & $1.90(0.076)$ & $3.55(0.056)$ & 1.65 & 2.03 & 3.48 & 1.45 \\
\hline SVWN5 & $0.62(0.042)$ & $2.18(0.029)$ & 1.56 & 0.72 & 2.19 & 1.47 \\
\hline BLYP & $0.49(0.060)$ & $2.03(0.040)$ & 1.54 & 0.61 & 2.07 & 1.46 \\
\hline B3LYP & $0.77(0.066)$ & $2.33(0.044)$ & 1.56 & 0.91 & 2.36 & 1.46 \\
\hline PBE & $0.59(0.087)$ & $2.14(0.064)$ & 1.55 & - & - & - \\
\hline PBE0 & $0.99(0.068)$ & $2.55(0.050)$ & 1.56 & - & - & - \\
\hline BHandHLYP & - & - & - & 1.32 & 2.77 & 1.45 \\
\hline $\operatorname{Exp}^{\mathrm{d}}$ & 1.14 & 2.66 & 1.52 & 1.14 & 2.66 & 1.52 \\
\hline
\end{tabular}

${ }^{a}$ Results obtained using the 4c-LR-KU-TDDFT method developed in this work. Data in the table represent mean values for each degenerate multiplet with the standard deviation in parenthesis.

b Data taken from Ref. 34

c $\triangle=J_{3 / 2}-J_{5 / 2}$ represents splitting of ${ }^{2} D\left[5 d^{9} 6 s^{2}\right]$ state.

d Data taken from NISC Atomic Spectra Database. ${ }^{60}$

TABLE VI. Low-lying excited states of $\mathrm{Rg}($ in $\mathrm{eV}) .^{\mathrm{a}}$

\begin{tabular}{lccc}
\hline \hline${ }^{2} D\left[7 s^{2} 6 d^{9}\right]$ & $J_{5 / 2}$ & $J_{3 / 2}$ & $\triangle^{\mathrm{b}}$ \\
\hline DHF & $0.00(0.012)$ & $2.80(0.024)$ & 2.80 \\
SVWN5 & $0.06(0.087)$ & $2.88(0.035)$ & 2.82 \\
BLYP & $-0.01(0.120)$ & $2.78(0.131)$ & 2.79 \\
B3LYP & $-0.20(0.165)$ & $2.53(0.154)$ & 2.74 \\
PBE & $-0.06(0.087)$ & $2.76(0.091)$ & 2.83 \\
PBE0 & $-0.20(0.159)$ & $2.53(0.163)$ & 2.73 \\
\hline${ }^{2} S\left[7 s 6 d^{10}\right]$ & $J_{1 / 2}$ & & \\
\hline DHF & $2.68(0.000)$ & & \\
SVWN5 & $2.79(0.046)$ & & \\
BLYP & $2.70(0.050)$ & & \\
B3LYP & $2.59(0.336)$ & & \\
PBE & $2.69(0.047)$ & & \\
PBE0 & $2.54(0.237)$ & & \\
\hline \hline
\end{tabular}

a Results obtained using the 4c-LR-KU-TDDFT method developed in this work. Data in the table represent mean values for each degenerate multiplet with the standard deviation in parenthesis.

b $\triangle=J_{3 / 2}-J_{5 / 2}$ represents splitting of ${ }^{2} D\left[7 s^{2} 6 d^{9}\right]$ state.

in calculations performed with the PBE, PBE0, BLYP, and B3LYP functionals. The same energies are swapped in $\mathrm{La}^{3+}$ calculations performed with $\mathrm{DHF}$ and the PBE, PBE0, and B3LYP functionals, and in the case of the BLYP functional the energy of the ${ }^{3} P_{2}$ level is predicted to be greater than those of both the ${ }^{3} F_{4}$ and ${ }^{3} F_{3}$ levels.

Atomic systems with a doubly degenerate ground state: For the first test of the proposed four-component linear response Kramers-unrestricted TDDFT method, 4c-LR-KU-TDDFT, on systems with a degenerate ground state we choose the calculation of the lowest excitation energies of Group 11 atoms $(\mathrm{Cu}-$ $\mathrm{Rg})$. Coinage-metal atoms $(\mathrm{Cu}-\mathrm{Au})$ are preferred for their relatively simple ground state electron configuration, ${ }^{2} S\left[(n-1) d^{10} n s, J_{1 / 2}\right]$, which results in smooth convergence of the self-consistent-field (SCF) procedure. Conversely, the ground state configuration of the $\mathrm{Rg}$ atom, ${ }^{2} D\left[7 s^{2} 6 d^{9}, J_{5 / 2}\right]$, differs from those of coinage-metal atoms, and the small HOMO-LUMO gap associated with this configuration results in slower SCF convergence.

A distinctive feature of the proposed 4c-LR-KUTDDFT method is that all states within a degenerate manifold are treated independently. For example, the first excitation energy for the gold atom is zero, since its ground state is doubly degenerate. This behavior is observed for all coinage-metal atoms, as the first excitation energy is always smaller then $10^{-4} \mathrm{eV}$. On the other hand, the sixfold degenerate ground state of the $\mathrm{Rg}$ atom is reproduced only approximately. Moreover, the reference state usually corresponds to a higher energy, which leads to negative average values. The worst results were those obtained with the B3LYP functional, which predicted $-0.20 \pm 0.17 \mathrm{eV}$ (see Table VI). This artificial energy splitting is inherent to Kramers-unrestricted methods that do not take into account the spatial symmetry of the system. This problem is not a consequence of the DFT functional used (since it is also present in DHF calculations), or of the eigensolver (since the artificial splitting is higher than the convergence threshold). In an attempt to quantify the significance of this issue we have calculated the mean and standard deviation of the energy excitations for each degenerate manifold, see Tables III-VII.

The DHF and DFT SCF calculations on the Rg atom predicted different ground state configurations, ${ }^{2} S\left[7 s 6 d^{10}, J_{1 / 2}\right]$ for the former and ${ }^{2} D\left[7 s^{2} 6 d^{9}, J_{5 / 2}\right]$ for the latter. However, the 4c-LR-KU-TDHF calculation converged to six negative energies, indicating that ${ }^{2} D\left[7 s^{2} 6 d^{9}, J_{5 / 2}\right]$ is the correct ground state configuration. DHF data in Table VI are presented relative to the ${ }^{2} D\left[7 s^{2} 6 d^{9}, J_{5 / 2}\right]$ configuration, so as to facilitate com- 
parison with the DFT results.

The convergence and number of iterations required for each energy level included in the calculation is found to be similar to that seen in calculations of excitation energies of systems with a non-degenerate ground state, $\mathrm{Sc}^{3+}-\mathrm{Ac}^{3+}$. In other words, the proposed EPS algorithm provides the same robust convergence for both Kramersrestricted and unrestricted reference states.

In Tables III-V we compare calculated and experimental low-lying excitation energies of coinage-metal atoms. These data were obtained using the 4c-LR-KU-TDDFT method developed in this work, and with the sf-X2C-STDA-SOC method presented in Ref. 34. The significant difference between these two sets of data is a consequence of the interplay between SOC and electron correlation. In the 4c-LR-KU-TDDFT results there are notable differences in the prediction of SO splitting, $\triangle$, obtained using different DFT functionals and DHF. On the other hand, the SO splitting is almost constant in the sf-X2C-S-TDASOC results, with the exception of the $\triangle=J_{3 / 2}-J_{5 / 2}$ splitting of $\mathrm{Ag}$, for which the DHF result deviates from this trend.

In $\mathrm{Cu}$ calculations with BLYP and B3LYP functionals, we obtained spurious negative eigenvalues, $-24.6 \mathrm{eV}$, $-24.6 \mathrm{eV}$, and $-23.4 \mathrm{eV}$. Under normal circumstances this would suggest that the SCF procedure converged to some stationary point instead of the ground state. However, these eigenvalues are unusually large and hence clearly unphysical. Another indication that they are artificial is that there is little difference in the structure and magnitude of PBE and BLYP one-electron eigenspectrum. Furthermore, the PBE calculations exhibit proper behavior and yield sensible results. We have therefore omitted these results in Table III and left their analysis to a future work.

The case of the $\mathrm{Ag}$ atom is especially challenging since the energy gap between states ${ }^{2} P\left[4 d^{10} 5 p, J_{3 / 2}\right]$ and ${ }^{2} D\left[4 d^{9} 5 s^{2}, J_{5 / 2}\right]$ is less then $0.1 \mathrm{eV}$, as indicated by the experimental data. Both the sf-X2C-S-TDA-SOC and 4c-LR-KU-TDDFT methods predict an incorrect ordering of the states. Use of the former method with the DHF Hamiltonian results in a large error in the SO splitting, whilst use of the 4c-LR-KU-TDDFT with the BLYP and B3LYP functionals mixes the ${ }^{2} P\left[4 d^{10} 5 p\right]$ states into the ${ }^{2} D\left[4 d^{9} 5 s^{2}\right]$ states, resulting in a large artificial splitting of the degenerate manifold. By increasing the threshold parameter, $\Theta$, from $10^{-16}$ to $10^{-15}$ results for BLYP and B3LYP functionals become 3.88(0.012) / 4.01(0.008) and $3.79(0.011) / 3.94(0.052)\left(J_{1 / 2} / J_{3 / 2}\right)$, respectively. Although the problem appears to be fixed, the first excitation energy becomes non-zero $\left(\sim 10^{-3}\right)$, which suggests that a less ad hoc solution should be sought, e.g., one which takes atomic symmetry into account.

Overall, the functional offering the best performance in the 4c-LR-KU-TDDFT method is PBE0, yielding slightly better results than the best obtained with the sfX2C-S-TDA-SOC method and BHandHLYP functional in the work of $\mathrm{Li}$ et al.. ${ }^{34}$ This is somewhat surprising
TABLE VII. Low-lying excited states of some actinide complexes $\left(\right.$ in $\mathrm{cm}^{-1}$ ).

\begin{tabular}{|c|c|c|c|c|}
\hline & & \multicolumn{3}{|c|}{${ }^{2} T_{2 u}$} \\
\hline & & $G_{3 / 2 u}$ & $E_{5 / 2 u}^{\prime}$ & $\triangle^{\mathrm{a}}$ \\
\hline \multirow[t]{8}{*}{$\mathrm{PaCl}_{6}^{2-}$} & $\mathrm{DHF}^{\mathrm{b}}$ & $999(672)$ & $5013(206)$ & 4014 \\
\hline & SVWN5 ${ }^{\mathrm{b}}$ & $2810(761)$ & $5018(284)$ & 2208 \\
\hline & $\mathrm{BLYP}^{\mathrm{b}}$ & 2946(367) & 4952(504) & 2006 \\
\hline & B3LYP ${ }^{b}$ & $2768(332)$ & $5028(447)$ & 2260 \\
\hline & $\mathrm{PBE}^{\mathrm{b}}$ & $2883(381)$ & $5067(469)$ & 2184 \\
\hline & $\mathrm{PBE}^{\mathrm{b}}$ & $2643(328)$ & $5211(377)$ & 2568 \\
\hline & SO-CASPT $2^{\mathrm{c}}$ & 2190 & 6000 & 3810 \\
\hline & $\operatorname{Exp}^{\mathrm{d}}$ & 2110 & 5250 & 3140 \\
\hline \multirow[t]{8}{*}{$\mathrm{UCl}_{6}^{-}$} & $\mathrm{DHF}^{\mathrm{b}}$ & $2984(683)$ & $6754(218)$ & 3770 \\
\hline & SVWN5 $5^{\mathrm{b}}$ & $4329(647)$ & $6327(201)$ & 1998 \\
\hline & BLYP $^{b}$ & $4691(322)$ & $6409(565)$ & 1718 \\
\hline & B3LYP ${ }^{b}$ & $4566(296)$ & $6572(490)$ & 2006 \\
\hline & $\mathrm{PBE}^{\mathrm{b}}$ & $4557(352)$ & $6419(511)$ & 1862 \\
\hline & $\mathrm{PBE}^{\mathrm{b}}$ & $4433(333)$ & $6706(432)$ & 2273 \\
\hline & SO-CASPT $2^{\mathrm{c}}$ & 3790 & 7300 & 3510 \\
\hline & $\operatorname{Exp}^{\mathrm{e}}$ & 3800 & 6790 & 2990 \\
\hline \multirow[t]{8}{*}{$\mathrm{NpF}_{6}$} & $\mathrm{DHF}^{\mathrm{b}}$ & $8297(141)$ & $9921(45)$ & 1624 \\
\hline & SVWN5 $5^{\mathrm{b}}$ & $8077(483)$ & $9183(86)$ & 1106 \\
\hline & $\mathrm{BLYP}^{\mathrm{b}}$ & $8871(235)$ & $9662(429)$ & 791 \\
\hline & B3LYP & 8683(191) & $9631(393)$ & 948 \\
\hline & $\mathrm{PBE}^{\mathrm{b}}$ & $8618(290)$ & $9458(398)$ & 840 \\
\hline & $\mathrm{PBE}^{\mathrm{b}}$ & $8458(266)$ & $9519(374)$ & 1061 \\
\hline & $\mathrm{SO}^{-\mathrm{CASPT}} 2^{\mathrm{c}}$ & 7280 & 9490 & 2210 \\
\hline & $\operatorname{Exp}^{f}$ & 7500 & 9400 & 1900 \\
\hline
\end{tabular}

a $\triangle=E_{5 / 2 u}^{\prime}-G_{3 / 2 u}$ represents splitting of ${ }^{2} T_{2 u}$ state.

b Results obtained using the 4c-LR-KU-TDDFT method developed in this work. Data in the table represent mean values for each degenerate multiplet with the standard deviation in parenthesis.

c Data taken from Ref. 62

d Data taken from Ref. 79

e Data taken from Ref. 80

f Data taken from Ref. 81

given that the sf-X2C-S-TDA-SOC method involves a number of approximations described above. However, as of yet there is too little data to draw any strong conclusions regarding the relative performance of the two methods.

Octahedral actinide complexes with a doubly 
degenerate groundstate: As a last test we chose three actinide complexes, $\mathrm{PaCl}_{6}^{2-}, \mathrm{UCl}_{6}^{-}$, and $\mathrm{NpF}_{6}$, from the work of Notter and Bolvin. ${ }^{62}$ This choice was motivated by the availability of experimental data for the spinorbit splitting of the lowest excitation state $\left({ }^{2} T_{2 u} \rightarrow\right.$ $\left.G_{3 / 2 u}, E_{5 / 2 u}^{\prime}\right)$. Furthermore, these systems are essentially single determinant in character, enabling comparison of our four-component TDDFT results with those obtained from post-Hartree-Fock methods which include spin-orbit effects (SO-CASPT2 in this case).

In contrast to Group 11 atoms, the first excitation energy in actinide complexes is not always well reproduced. For $\mathrm{NpF}_{6}, \mathrm{UCl}_{6}^{-}$, and $\mathrm{PaCl}_{6}^{2-}$ the first excitation energies are always smaller than $10 \mathrm{~cm}^{-1}, 100 \mathrm{~cm}^{-1}$, and $200 \mathrm{~cm}^{-1}$, respectively. Although this behavior is not ideal, it is consistent with the energy spread (quantified by the standard deviation, see Table VII) of the energy manifold of states $G_{3 / 2 u}$ and $E_{5 / 2 u}^{\prime}$.

The convergence behavior of the EPS algorithm is in most cases similar to previous calculations, requiring $\sim 10$ matrix-vector contractions per each energy level. Deviations from this trend were observed in only a few cases (all of which used hybrid functionals) where up to 20 contractions were necessary to converge one energy level, and in all DHF calculations, where more then 30 contractions were required. In addition, in the case of a DHF calculation of the $\mathrm{PaCl}_{6}^{2-}$ system the first excitation energy $\left(\sim 10^{-3} \mathrm{au}\right)$ was calculated to have a large imaginary component, suggesting an issue with the reference wave function rather than merely inefficiency of the eigenproblem solver. However, solution of this issue is left for future studies.

Two conclusions can be drawn from the results for DFT functionals in Table VII. First, among all DFT functionals PBE0 performs best when compared to experimental data. Second, all DFT results are better for the $E_{5 / 2 u}^{\prime}$ than the $G_{3 / 2 u}$ state, and errors in the spinorbit driven energy splitting are mostly caused by overestimation of the energy of the $G_{3 / 2 u}$ state. Overall, the PBE0 results were comparable to those obtained using SO-CASPT2 for all states, indicating the suitability of DFT for the prediction of the energy spectra of heavyatom containing systems with degenerate ground states.

\section{SUMMARY AND CONCLUDING REMARKS}

In this work we have reported a method for prediction of the excitation spectra of heavy-atom containing molecules with both non-degenerate and degenerate ground states. The proposed 4c-LR-KU-TDDFT method uses a four-component Kramers-unrestricted noncollinear DFT methodology, and is therefore suitable for treatment of relativistic effects, regardless of their strength, across the periodic table of elements. This development required two major improvements to existing relativistic TDDFT methodologies.

First, the DFT potential and kernel resulting from the
Scalmani and Frisch noncollinear ansat $z^{44}$ has been regularized so as to ensure correct behavior in limit cases involving small spin densities and small spin density gradients. The regularization was chosen so as to reproduce the results obtained from non-relativistic DFT functionals in the closed shell limit, i.e., in cases where $\vec{\rho} \rightarrow \rho_{z}$ and $\vec{\nabla} \vec{\rho} \rightarrow \vec{\nabla} \rho_{z}$. In the limit case where the spin density and spin density gradients of the reference state are vanishingly small, we chose to reproduce results obtained using the well-behaved noncollinear DFT kernel described by Bast et $a .^{32}$. The proposed noncollinear DFT potential and kernel depend on an arbitrary parameter, $\Theta$, which should be considered as part of their definition. The resulting noncollinear methodology is applicable to both Kramers-restricted and unrestricted reference states, i.e., the proposed noncollinear DFT potential and kernel is suitable for states with both zero and non-zero magnetization.

The second improvement was to the Davidson-Olsen iterative subspace algorithm ${ }^{56,57}$ intended to solve those TDDFT eigenvalue problems for which the size of the relevant matrix prohibits direct diagonalization. The distinguishing feature of the modification is the use of left eigenvectors in construction of the trial subspace. This improvement was found to be essential to ensure the robust convergence of the TDDFT non-Hermitian eigenproblem for both Kramers-restricted and unrestricted reference states. The modified algorithm has enabled calculations of up to the first one hundred eigenvalues.

We have demonstrated the capability of the presented method to calculate vertical excitation energies of systems with non-degenerate ground states, $\mathrm{Sc}^{3+}{ }_{-} \mathrm{Ac}^{3+}$, and doubly degenerate ground states, $\mathrm{Cu}-\mathrm{Rg}, \mathrm{PaCl}_{6}^{2-}, \mathrm{UCl}_{6}^{-}$, and $\mathrm{NpF}_{6}$. Overall, the presented 4c-LR-KU-TDDFT method yields best results when used in conjunction with the PBE0 functional. The performance of the 4c-LR-KUTDDFT method presented here suggests the suitability of DFT for prediction of energy spectra of heavy-atom containing systems with both non-degenerate and doubly degenerate ground states.

\section{ACKNOWLEDGMENTS}

Financial support from the Slovak Grant Agencies VEGA and APVV (contract no. 2/0116/17 and APVV15-0726), and financial support from the SASPRO Program (Contract No. 1563/03/02), co-financed by the European Union and the Slovak Academy of Sciences, is duly acknowledged. We are also grateful for support from the National Scholarship Programme of the Slovak Republic funded by the Ministry of Education, Science, Research and Sport of the Slovak Republic (id 24522). This work has also received support from Research Council of Norway through a Center of Excellence Grant (Grant No. 262695). Computational resources have been provided by the Norwegian Supercomputer Program NOTUR (Grant No. NN4654K). We are thankful to Michael Patzschke 
and Hans Jørgen Aagaard Jensen for valuable discussion.

${ }^{1}$ M. E. Casida, in Recent Advances in Density Functional Methods, Part I, edited by D. P. Chong (World Scientific, Singapore, 1995) Chap. 5, pp. 155-192.

${ }^{2}$ M. L. Marques and E. K. U. Gross, Annu. Rev. Phys. Chem. 55 427 (2004).

${ }^{3}$ B. K., J. Werschnik, and E. K. U. Gross, J. Chem. Phys. 123 062206 (2005).

${ }^{4}$ A. Dreuw and M. Head-Gordon, Chem. Rev. 105, 4009 (2005).

${ }^{5}$ P. Elliott, F. Furche, and K. Burke, in Reviews in Computational Chemistry Vol. 26, edited by K. B. Lipkowitz, T. R. Cundari, and D. B. Boyd (Wiley, New Jersey, U.S.A., 2009) Chap. 3, pp. 91-165.

${ }^{6}$ M. E. Casida, J. Mol. Struct. (Theochem) 914, 3 (2009).

${ }^{7}$ A. L. M. Marques, N. T. Maitra, F. M. S. Nogueira, E. K. U. Gross, and A. Rubio, Fundamentals of Time-Dependent Density-Functional-Theory (Springer-Verlag Berlin Heidelberg, 2012)

${ }^{8}$ M. E. Casida and M. Huix-Rotllant, Annu. Rev. Phys. Chem. 63, 287 (2012)

${ }^{9}$ A. D. Laurent and D. Jacquemin, Int. J. Quantum Chem. 113, 2019 (2013)

${ }^{10}$ F. de Groot, Chem. Rev. 101, 1779 (2001)

${ }^{11}$ R. Boča, Coord. Chem. Rev, 248, 757 (2004).

${ }^{12}$ R. C. Evans, P. Douglas, and C. J. Winscom, Coord. Chem. Rev. 250, 2093 (2006).

13 A. Köhler and H. Bässler, Mater. Sci. Eng. R Rep. 66, 71 (2009). ${ }^{14}$ Q. Zhao, F. Li, and C. Huang, Chem. Soc. Rev 39, 3007 (2010). ${ }^{15}$ S. Sanvito, Chem. Soc. Rev 40, 3336 (2011).

${ }^{16}$ N. Nagaosa and Y. Tokura, Nat. Nanotechnol. 8, 899 (2013).

${ }^{17}$ G. Baryshnikov, B. Minaev, and H. Ågren, Chem. Rev. 117, 6500 (2017)

${ }^{18}$ M. Kühn and F. Weigend, J. Chem. Phys. 141, 224302 (2014)

${ }^{19}$ K. Mori, T. P. M. Goumans, E. van Lenthe, and F. Wang, Phys. Chem. Chem. Phys. 28, 14523 (2014).

${ }^{20}$ L. Jeremias, J. Novotný, M. Repisky, S. Komorovsky, and R. Marek, Inorg. Chem. 57, 8748 (2018).

${ }^{21}$ J. Vícha, S. Komorovsky, M. Repisky, R. Marek, and M. Straka, J. Chem. Theory Comp. 14, 3025 (2018).

${ }^{22}$ T. B. Demissie, J. Chem. Phys. 147, 174301 (2017).

${ }^{23}$ J. Vícha, R. Marek, and M. Straka, Inorg. Chem. 55, 10302 (2016).

${ }^{24}$ S. Gohr, P. Hrobárik, M. Repiský, S. Komorovský, K. Ruud, and M. Kaupp, J. Phys. Chem. A 119, 12892 (2015).

${ }^{25}$ N. T. Maitra, K. Burke, H. Appel, E. K. U. Gross, and R. van Leeuwen, in Reviews of Modern Quantum Chemistry: A Celebration of the Contributions of Robert G. Parr, edited by K. D. Sen (World-Scientific, Singapore, 2002) pp. 1186-1225.

${ }^{26}$ T. Ando, Z. Phys. B 26, 263 (1977).

${ }^{27}$ T. Ando, Solid State Commun. 21, 133 (1977)

${ }^{28}$ S. Hirata and M. Head-Gordon, Chem. Phys. Lett. 314, 291 (1999).

${ }^{29}$ J. Gao, W. Zou, W. Liu, Y. Xiao, D. Peng, B. Song, and C. Liu, J. Chem. Phys. 123, 054102 (2005).

${ }^{30}$ F. Wang and T. Ziegler, J. Chem. Phys. 122, 204103 (2005).

${ }^{31}$ W. Liu and Y. Xiao, Chem. Soc. Rev 47, 4481 (2018).

${ }^{32}$ R. Bast, H. J. A. Jensen, and T. Saue, Int. J. Quantum Chem. 109, 2091 (2009)

${ }^{33}$ M. Kühn and F. Weigend, J. Chem. Theory Comp. 9, 5341 (2013).

${ }^{34}$ Z. Li, B. Suo, Y. Zhang, Y. Xiao, and W. Liu, Mol. Phys. 111 3741 (2013).

${ }^{35}$ F. Egidi, S. Sun, J. J. Goings, G. Scalmani, M. J. Frisch, and X. Li, J. Chem. Theory Comp. 13, 2591 (2017).

${ }^{36}$ F. Réal, V. Vallet, C. Marian, and U. Wahlgren, J. Chem. Phys. 127, 214302 (2007)

${ }^{37}$ K. Pierloot and E. Van Besien, J. Chem. Phys. 123, 204309 (2005).
${ }^{38}$ P. Tecmer, A. S. P. Gomes, U. Ekström, and L. Visscher, Phys. Chem. Chem. Phys. 13, 6249 (2011).

${ }^{39}$ R. G. Denning, T. R. Snellgrove, and D. R. Woodward, Mol. Phys. 37, 1109 (1979).

${ }^{40}$ F. G. Eich and E. K. U. Gross, Phys. Rev. Lett. 111, 156401 (2013).

${ }^{41}$ F. G. Eich, S. Pittalis, and G. Vignale, Phys. Rev. B 88, 245102 (2013)

${ }^{42}$ D. Gontier, Phys. Rev. Lett. 111, 153001 (2013)

${ }^{43}$ E. I. Tellgren, Phys. Rev. A 97, 022513 (2018).

${ }^{44}$ G. Scalmani and M. J. Frisch, J. Chem. Theory Comp. 8, 2193 (2012).

45 J. Kubler, K. H.hock, J. Sticht, and A. Williams, 18, 469 (1988)

${ }^{46}$ L. M. Sandratskii, Adv. Phys. 47, 91 (1998).

${ }^{47}$ C. van Wüllen, J. Comput. Chem. 23, 779 (2002).

${ }^{48}$ A. Petrone, D. B. Williams-Young, S. Sun, T. F. Stetina, and X. Li, Eur. Phys. J. B 91, 169 (2018).

${ }^{49}$ F. Wang and T. Ziegler, J. Chem. Phys. 121, 12191 (2004).

${ }^{50}$ R. E. Stanton and S. Havriliak, J. Chem. Phys. 81, 1910 (1984)

${ }^{51}$ W. Kutzelnigg, Int. J. Quantum Chem. 25, 107 (1984).

${ }^{52}$ L. Visscher and K. G. Dyall, At. Data Nucl. Data Tables 67, 207 (1997).

${ }^{53}$ The nabla operator, $\vec{\nabla}$, in the charge and spin density gradients acts only on the density itself not on the following functions.

${ }^{54}$ J. Peralta, G. Scuseria, and M. Frisch, Phys. Rev. B Condens. Matter 75, 125119 (2007).

${ }^{55}$ S. Komorovsky, M. Repisky, E. Malkin, T. B. Demissie, and K. Ruud, J. Chem. Theory Comp. 11, 3729 (2015).

${ }^{56}$ E. R. Davidson, J. Comput. Phys. 17, 87 (1975)

${ }^{57}$ J. Olsen, P. Jørgensen, and J. Simons, Chem. Phys. Lett. 169 463 (1990).

${ }^{58}$ J. Olsen, H. J. A. Jensen, and P. Jørgensen, J. Comput. Phys. 74, 265 (1988)

${ }^{59}$ F. Egidi, J. J. Goings, M. J. Frisch, and X. Li, J. Chem. Theory Comp. 12, 3711 (2016).

${ }^{60}$ A. Kramida; Y. Ralchenko; J. Reader and NIST ASD Team (2018). NIST Atomic Spectra Database (version 5.5.6), [Online]. Available: https://physics.nist.gov/asd [accessed Aug 09, 2018]. National Institute of Standards and Technology, Gaithersburg, MD.

${ }^{61}$ ReSpect, version 5.1.0 (2019) - Relativistic Spectroscopy DFT program of authors S. Komorovsky; M. Repisky; V. G. Malkin O. L. Malkina; M. Kaupp; K. Ruud, with contributions from R. Bast; U. Ekström; M. Hrdá; M. Kadek; S. Knecht; L. Konecny; A. Křístková; E. Malkin; I. Malkin-Ondik; R. Di Remigio. See www.respectprogram.org, (accessed July 26, 2019).

${ }^{62}$ F. P. Notter and H. Bolvin, J. Chem. Phys. 130, 184310 (2009)

${ }^{63}$ S. H. Vosko, L. Wilk, and M. Nusair, Canad. J. Phys. 58, 1200 (1980).

${ }^{64}$ A. D. Becke, Phys. Rev. A 38, 3098 (1988).

${ }^{65}$ C. Lee, W. Yang, and R. Parr, Phys. Rev. B 37, 785 (1988).

${ }^{66}$ P. J. Stephens, F. J. Devlin, C. F. Chabalowski, and M. J. Frisch, J. Phys. Chem. 98, 11623 (1994).

${ }^{67}$ J. P. Perdew, K. Burke, and M. Ernzerhof, Phys. Rev. Lett. 77, 3865 (1996).

${ }^{68}$ J. P. Perdew, K. Burke, and M. Ernzerhof, Phys. Rev. Lett. 78 1396 (1997).

${ }^{69} \mathrm{C}$. Adamo and V. Barone, J. Chem. Phys. 110, 6158 (1999).

${ }^{70}$ U. Ekström, L. Visscher, R. Bast, A. J. Thorvaldsen, and K. Ruud, J. Chem. Theory Comp. 6, 1971 (2010).

${ }^{71} \mathrm{~K}$. G. Dyall, Basis sets for $3 \mathrm{p}$ and 3d Elements are available on the web site http://dirac.chem.sdu.dk/basisarchives/dyall.

72 K. G. Dyall, Theor. Chem. Acc. 117, 483 (2007).

${ }^{73}$ K. G. Dyall, Theor. Chem. Acc. 112, 403 (2004).

${ }^{74}$ K. G. Dyall and A. S. P. Gomes, Theor. Chem. Acc. 125, 97 (2010).

${ }^{75}$ K. G. Dyall, Theor. Chem. Acc. 129, 603 (2011).

${ }^{76}$ K. G. Dyall, Theor. Chem. Acc. 117, 491 (2007).

${ }^{77}$ J. C. Boettger, Phys. Rev. B 62, 7809 (2000).

${ }^{78} \mathrm{~F}$. Neese, in Calculation of NMR and EPR Parameters. Theory 
and Applications, edited by M. Kaupp, M. Bühl, and V. G. Malkin (Wiley-VCH, Weinheim, 2004) Chap. 34, pp. 541-564. ${ }^{79}$ N. Edelstein, D. Brown, and B. Whittaker, Inorg. Chem. 13,
563 (1974).

${ }^{80}$ J. Selbin, J. D. Ortego, and G. Gritzner, Inorg. Chem. 7, 976 (1968).

${ }^{81}$ G. L. Goodman and M. Fred, J. Chem. Phys. 30, 849 (1959). 